\title{
REGULARITY OF SOLUTIONS OF PARABOLIC EQUATIONS WITH A DOUBLE NONLINEARITY AND A WEIGHT
}

\author{
M. D. SURNACHËV \\ Dedicated to the centenary of the great mathematician \\ Boris Moiseevich Levitan
}

\begin{abstract}
We study local regularity of solutions of nonlinear parabolic equations with a double degeneracy and a weight. We impose the condition of $p$-admissibility on the weight; in particular this allows weights in the Muckenhoupt classes $A_{p}$. We prove that solutions are locally Hölderian without any restriction on the sign being constant. We prove a Harnack inequality for nonnegative solutions. We examine the stability of the constants as the parameters in the equation approach the linear case.
\end{abstract}

\section{$\S 1$. INTRODUCTION}

In this paper we study the regularity of solutions of nonlinear parabolic equations of the form

$$
\nu(x) u_{t}=\operatorname{div}(\nu(x) \mathbf{A}(x, t, u, \nabla u)), \quad x \in \Omega \subset \mathbb{R}^{n}, t \in\left[T_{1}, T_{2}\right] .
$$

It is assumed that the flow

$$
\mathbf{A}(x, t, u, \xi): \Omega \times\left[T_{1}, T_{2}\right] \times \mathbb{R} \times \mathbb{R}^{n} \rightarrow \mathbb{R}^{n}
$$

is a Carathéodory function (that is, measurable with respect to $(x, t)$ for all $(u, \xi)$ and continuous with respect to $(u, \xi)$ for almost all $(x, t))$ satisfying the conditions

$$
\begin{gathered}
|\mathbf{A}(x, t, u, \xi)| \leq C_{0}|u|^{m-1}|\xi|^{p-1}+C_{u}, \\
\mathbf{A}(x, t, u, \xi) \cdot \xi \geq C_{1}|\xi|^{p}|u|^{m-1}-C_{l},
\end{gathered}
$$

where $p>2$ and $m>1$ are constants, for all $(x, t) \in \mathbb{R}^{n} \times\left[T_{1}, T_{2}\right]$ and $u \in \mathbb{R}, \xi \in \mathbb{R}^{n}$; here $C_{0}, C_{1}$ are positive constants and $C_{u}, C_{l}$ are nonnegative constants.

The weight $\nu=\nu(x)$ is a nonnegative function in $L_{\text {loc }}^{1}\left(\mathbb{R}^{n}\right)$. We use the same letter $\nu$ to denote the measure associated with the weight $\nu$; that is, $d \nu=\nu d x$ and $\nu(A)=\int_{A} \nu d x$, $\nu(B)=\int_{B} \nu d x d t$ for measurable sets $A \subset \mathbb{R}^{n}$ and $B \subset \mathbb{R}^{n+1}$.

We assume that the weight $\nu$ satisfies the condition of $p$-admissibility:

I. (Doubling): There exists a constant $C_{2}>0$ such that

$$
\nu(2 B) \leq C_{2} \nu(B)
$$

for any ball $B \subset \mathbb{R}^{n}$. Here $2 B$ is the ball with the same centre as $B$ but with doubled radius.

2010 Mathematics Subject Classification. Primary 35K92; Secondary 35K65.

Key words and phrases. Nonlinear parabolic equations, admissible weights, regularity of solutions, double degeneration, Muckenhoupt classes, Harnack inequality.

This research was supported by the Russian Foundation for Basic Research (grants No. 12-01-00058-a and 14-01-31341). 
II. (Uniqueness of the gradient): If $\varphi_{i}$ is a sequence of locally Lipschitz functions defined in the domain $\Omega \subset \mathbb{R}^{n}$ and such that

$$
\int_{\Omega}\left|\varphi_{i}\right|^{p} \nu d x \rightarrow 0 \quad \text { and } \quad \int_{\Omega}\left|\nabla \varphi_{i}-v\right|^{p} \nu d x \rightarrow 0
$$

as $i \rightarrow \infty$, where $v \in\left(L^{p}(\Omega ; \nu d x)\right)^{n}$, then $v \equiv 0$. This property guarantees that the gradient of the function is well defined.

III. (Sobolev's inequality): There exist constants $\kappa>1$ and $C_{3}>0$ such that

$$
\left(\frac{1}{\nu(B)} \int_{B}|\varphi|^{\kappa p} d \nu\right)^{1 /(\kappa p)} \leq C_{3} r\left(\frac{1}{\nu(B)} \int_{B}|\nabla \varphi|^{p} d \nu\right)^{1 / p}
$$

for any ball $B$ of radius $r$ in $\mathbb{R}^{n}$ and a Lipschitz function $\varphi$ with compact support in $B$.

IV. (Poincaré's inequality): There exists a constant $C_{4}>0$ such that

$$
\left(\frac{1}{\nu(B)} \int\left|\varphi-\varphi_{B}\right|^{p} \nu d x\right)^{1 / p} \leq C_{4} r\left(\frac{1}{\nu(B)} \int_{B}|\nabla \varphi|^{p} \nu d x\right)^{1 / p},
$$

where

$$
\varphi_{B}=\frac{1}{\nu(B)} \int_{B} \varphi d \nu
$$

for any ball $B$ of radius $r$ in $\mathbb{R}^{n}$ and a Lipschitz function $\varphi$.

It is also possible to study weighted Sobolev spaces without assuming the uniqueness of the gradient. Results in this direction were obtained in [9].

Two classical examples of $p$-admissible weights are given by the Muckenhoupt classes $A_{p}$ and expressions $[J(x)]^{1-p / n}$, where $J(x)$ is the Jacobian of a quasiconformal map for $1<p<n$. For example, the weight $|x|^{\delta}$ is $p$-admissible if $\delta>-n$. Recall that a weight $\nu$ belongs to a Muckenhoupt class $A_{p}$ if

$$
C_{p}(\nu)=\sup \left(\frac{1}{|K|} \int_{K} \nu(x) d x\right)\left(\frac{1}{|K|} \int_{K}\left(\frac{1}{\nu(x)}\right)^{1 /(p-1)} d x\right)^{p-1}<+\infty,
$$

where the supremum is taken over all cubes $K \subset \mathbb{R}^{n}$ with faces parallel to the coordinate planes. A map $f(x): \Omega \subset \mathbb{R}^{n} \rightarrow \mathbb{R}^{n}$ is said to be quasiconformal if $|D f(x)|^{n} \leq C\left|J_{f}(x)\right|$, where $J_{f}(x)=\operatorname{Det}[D f]$. Another source of $p$-admissible weights is provided by powers of weights in strong $A_{\infty}$ (see [21]). All Jacobians of quasiconformal maps are strong $A_{\infty}$ weights.

A crucially important property of $p$-admissible weights is the self-improving property established in 22]: if a weight is $p$-admissible, then it is also $(p-\varepsilon)$-admissible quantitatively; that is, all the constants in the definition of $(p-\varepsilon)$-admissibility and the number $\varepsilon>0$ are calculated in terms of the constants in the definition of $p$-admissibility. In this regard, $p$-admissible weights behave in the same way as Muckenhoupt weights: it is well known that if a weight belongs to a Muckenhoupt class $A_{p}, p>1$, then it also belongs to $A_{p-\varepsilon}$. Furthermore, the range of admissible $\varepsilon$ and the corresponding constant $C_{p-\varepsilon}(\nu)$ in the definition of a Muckenhoupt weight in $A_{p-\varepsilon}$ are expressed in terms of the original $A_{p}$-constant $C_{p}(\nu)$. Recall also that if a weight is $p$-admissible, then it is also $q$-admissible for any $q>p$.

The gradient $\nabla u$ and the weighted Sobolev space $W^{1, p}(\Omega ; \nu)$ are defined using the closure procedure: we say that for $u \in W^{1, p}(\Omega ; \nu)$ a function $v \in L^{p}(\Omega ; \nu)$ is the gradient of $u$, denoted by $v=\nabla u$, if $u \in L^{p}(\Omega ; \nu)$ and

$$
\int_{\Omega}\left|\varphi_{i}-u\right|^{p} d \nu+\int_{\Omega}\left|\nabla \varphi_{i}-v\right|^{p} d \nu \rightarrow 0 \quad \text { as } i \rightarrow \infty
$$


for some sequence of locally Lipschitz functions $\varphi_{i}$ on $\Omega$. The norm in $W^{1, p}(\Omega ; \nu)$ is defined as

$$
\|u\|_{W^{1, p}(\Omega ; \nu)}=\|u\|_{L^{p}(\Omega ; \nu)}+\|\nabla u\|_{L^{p}(\Omega ; \nu)} .
$$

For $p$-admissible weights, the gradient defined in this manner coincides with the ordinary gradient on locally Lipschitz functions. If a weight $\nu$ belongs to a Muckenhoupt class $A_{p}$, then it is easy to verify that the gradient of a function in $W^{1, p}(\Omega ; \nu)$ belongs to $L^{1}(\Omega)$ and coincides with the gradient of this function in the sense of distribution theory. The space $W_{0}^{1, p}(\Omega ; \nu)$ is defined as the closure of $C_{0}^{\infty}(\Omega)$ in $W^{1, p}(\Omega ; \nu)$.

The definition of a (weak) solution of equation (1.1) is standard: we say that $u$ is a solution of equation (1.1) in the cylinder $\mathcal{Q}:=\Omega \times\left[T_{1}, T_{2}\right]$ if

$$
u \in C\left(\left[T_{1}, T_{2}\right] ; L^{2}(\Omega ; \nu)\right), \quad\left(1+|u|^{(m-1) p /(p-1)}\right)|\nabla u|^{p} \in L^{1}(\mathcal{Q} ; \nu),
$$

and

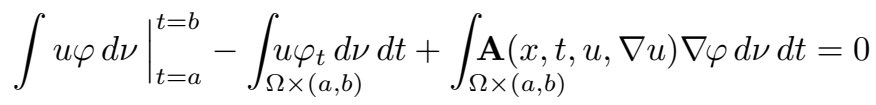

for any $[a, b] \subset\left[T_{1}, T_{2}\right]$ and $\varphi \in W^{1,1}\left(T_{1}, T_{2} ; L^{2}(\Omega ; \nu)\right) \cap L^{p}\left(T_{1}, T_{2} ; W_{0}^{1, p}(\Omega ; \nu)\right)$. The definition of a sub(super)solution of equation (1.1) differs in that a test function $\varphi$ is taken to be nonnegative, and instead of equality in (1.8), we have the sign $\geq$ for supersolutions and $\leq$ for subsolutions.

Equations with a double nonlinearity of the form (1.1) in the case when there is no weight, $\nu(x) \equiv 1$, were studied in a fair amount of detail by Ivanov in the cycle of papers $[1+8]$. Note that Ivanov studied sign-constant solutions, although the technique that he applied also works in the general case. In particular, he established local estimates for the maximum of the modulus of solutions and also the Hölder continuity of solutions. Studies in the same direction were conducted in parallel by Vespri 25 27.

Linear parabolic equations with weight were studied in [12 16. For nonlinear parabolic equations of $p$-Laplacian type with a Muckenhoupt weight, the Hölder property of solutions was obtained in [23, and a Harnack inequality was obtained in the author's paper 24. For equations with double nonlinearity with a Muckenhoupt weight, the Hölder continuity of solutions was studied in 11 .

We should point out that, in the main, two cases are considered. In the first the weight occurs only under the divergence sign. The model equation is of the form

$$
u_{t}=\operatorname{div}\left(\nu(x)|\nabla u|^{p-2} \nabla u\right) .
$$

Here, in the scale of Muckenhoupt classes, the requirement on the weight is $\nu \in A_{1+p / n}$. The papers [11,23, 24] are devoted to this case, and the results for the linear case which preceded these can be found in $[12,14,16$. In the last two papers weights depending on time were considered, $\nu=\nu(x, t)$.

The equations we consider in this paper are in the second class of equations, that is, the weight both multiplies the time derivative and lies under the divergence sign:

$$
\nu(x) u_{t}=\operatorname{div}\left(\nu(x)|\nabla u|^{p-2} \nabla u\right) .
$$

In this case, in the scale of Muckenhoupt classes, the class of admissible weights is wider, $\nu \in A_{p}$, and coincides with the class of admissible Muckenhoupt weights for elliptic equations. In the linear case, equations of this type were studied in 15 .

Using the classical Moser iteration it is easy to show that all solutions of (1.1) are locally bounded. We shall not give a proof of this here. In what follows we assume that all the solutions with which we work are a priori bounded. 
Notation. We shall need the following notation. We let $B_{R}$ denote the ball of radius $R$ with centre at the origin and $x_{0}+B_{R}$ denote the ball of radius $R$ with centre at $x_{0}$. For cylinders we introduce the notation

$$
\begin{gathered}
Q(R, T)=B_{R} \times[-T, 0], \\
\left(x_{0}, t_{0}\right)+Q(R, T)=\left\{(x, t):\left|x-x_{0}\right|<R, t_{0}-T \leq T \leq t_{0}\right\} .
\end{gathered}
$$

It is convenient to introduce the quantities

$$
l=\frac{m-1}{p} \quad \text { and } \quad h=1-\frac{1}{\kappa},
$$

where $\kappa>1$ is the constant in Sobolev's inequality (1.5). We say that a quantity depends on the parameters of the equation and write $C=C$ (data) if this quantity depends only on $n, p, m$ and the constants $C_{0-4}, C_{u}, C_{l}$. We use the symbols $C$ and $\gamma$ to denote various constants. Their values may be different in different places. We introduce the functions

$$
\theta(T, \mu, \omega, R)=\frac{T}{(\max (|\mu|, \omega))^{1-m} \omega^{2-p} R^{p}}
$$

and

$$
\Gamma(\mu, \omega, R)=\left(C_{l}+C_{u} \frac{\omega}{R}\right)\left(\frac{\omega}{R}\right)^{-p}(\max (|\mu|, \omega))^{1-m} .
$$

In the case of a homogeneous equation $\left(C_{u}=C_{l}=0\right)$, the quantity $\Gamma$ is identically zero. For a cylinder $Q$ the base of which is a ball of radius $R$, we use the notation

$$
T_{ \pm}(u, Q)=\left(\max \left(\left|\mu_{ \pm}\right|, \omega\right)\right)^{1-m} \omega^{2-p} R^{p},
$$

where

$$
\mu_{+}=\operatorname{ess} \sup \{u ; Q\}, \quad \mu_{-}=\operatorname{ess} \inf \{u ; Q\}, \quad \omega=\frac{\mu_{+}-\mu_{-}}{2} .
$$

For a sign-changing quantity $k$ the expression $k^{1+l}$ is understood as $|k|^{l} k$.

Theorem 1. Let $u$ be a solution of (1.1) in the cylinder $\mathcal{Q}=\Omega \times\left[T_{1}, T_{2}\right]$ such that $\operatorname{ess} \sup \{u ; \mathcal{Q}\}=M<\infty$. Then, up to changes on a set of measure zero, the function $u$ is locally Hölder continuous, and its modulus of continuity in the inner subcylinder $\Omega_{1} \times\left(T_{1}+\varepsilon, T_{2}\right), \Omega_{1} \Subset \Omega, \varepsilon>0$, depends only on the data of the equation, $M, \varepsilon$, and the distance from $\Omega_{1}$ to the boundary of $\Omega$.

In the following theorem, we assume without loss of generality that the solution under consideration is continuous.

Theorem 2. Suppose that a nonnegative bounded solution of equation (1.1) is given in a cylinder $\mathcal{Q}=\left(x_{0}, t_{0}\right)+B_{17 R} \times\left[-\lambda T_{1}, T\right], \lambda>0$. Suppose that $u\left(x_{0}, t_{0}\right) \geq k>0$. Then there exist positive constants $\gamma_{i}=\gamma_{i}$ (data, $\left.\lambda\right), i=1,2,3$, such that if $T_{1}>k^{3-m-p} R^{p}$, $T \geq \gamma_{1} k^{3-p-m} R^{p}$, and $\Gamma(0, k, R) \leq \gamma_{2}$, then

$$
u\left(x, t_{0}+\gamma_{1} k^{3-p-m} R^{p}\right) \geq \gamma_{3} k, \quad\left|x-x_{0}\right|<R .
$$

Remark. The estimates of Theorems 1, 2 are stable as $p \rightarrow 2, m \rightarrow 1$.

\section{$\S 2$. Properties of weights and weighted Sobolev spaces}

It is well known that the conditions of elliptic $p$-admissibility given above can be reduced to just two conditions: the doubling condition I and Poincaré's $(1, p)$-inequality. For a ball $B$ in $\mathbb{R}^{n}$, let $\lambda B$ denote the ball with the same centre and with radius equal to $\lambda$ times the radius of $B$. 
Poincaré's $(1, p)$-inequality: For some constants $\lambda \geq 1$ and $C_{5}>0$, the inequality

$$
\frac{1}{\nu(B)} \int\left|\varphi-\varphi_{B}\right| \nu d x \leq C_{5} r\left(\frac{1}{\nu(\lambda B)} \int_{\lambda B}|\nabla \varphi|^{p} \nu d x\right)^{1 / p}
$$

holds for any ball $B \subset \mathbb{R}^{n}$ and a bounded locally Lipschitz function $\varphi: \lambda B \rightarrow \mathbb{R}$, where $r$ is the radius of $B$. It is known that if the weight satisfies the doubling condition and (2.1) with some $\lambda>1$, then (2.1) also holds with $\lambda=1$ (at the expense of changing $C_{5}$ ).

Obviously, Poincaré's $(1, p)$-inequality is a consequence of IV. On the other hand, any weight which satisfies the doubling condition and Poincaré's $(1, p)$-inequality also satisfies all the conditions of $p$-admissibility, and the constants in I-IV can be expressed in terms of the doubling constant in I and the constants in Poincaré's $(1, p)$-inequality.

Lebesgue's theorem on differentiability is also important to us:

$$
\lim _{r \rightarrow 0} \frac{1}{\nu(B(x, r))} \int_{B(x, r)} f d \nu=f(x)
$$

for any $f \in L_{\text {loc }}^{1}\left(\mathbb{R}^{n} ; \nu\right)$ and for almost all $x \in \mathbb{R}^{n}$. The following fact is a consequence of the doubling property; we present it with its proof (see [17]).

Lemma 3. For any ball $B=x_{0}+B_{r}$ and any $\sigma \in(0,1)$ we have

$$
\frac{\nu(B \backslash(1-\sigma) B)}{\nu(B)} \leq C \sigma^{\alpha}, \quad(1-\sigma) B=x_{0}+B_{(1-\sigma) r},
$$

where the positive constants $\alpha, C$ depend only on the doubling constant of the weight $\nu$ and the dimension of the space $n$.

Proof. Let $r_{j}=r\left(1-2^{-j}\right)$ and $B_{j}=x_{0}+B_{r_{j}}$. Consider the spherical layers $L_{i}=B \backslash B_{j}$. It follows from the doubling property for $\nu$ that $\nu\left(L_{i} \backslash L_{i+1}\right) \geq \delta \nu\left(L_{i+1}\right)$, where $\delta>0$. Hence, $\nu\left(L_{i}\right) \geq(1+\delta) \nu\left(L_{i+1}\right)$. Iterating we obtain

$$
\nu\left(L_{j}\right) \leq(1+\delta)^{1-j} \nu\left(L_{1}\right)<(1+\delta)^{-j} \nu(B) .
$$

It is now easy to see that

$$
\frac{\nu(B \backslash(1-\sigma) B)}{\nu(B)} \leq(1+\delta)^{\left[\log _{2} \sigma\right]} \leq(1+\delta)^{\log _{2} \sigma+1}=(1+\delta) \sigma^{\alpha},
$$

where $\alpha=1 / \log _{2}(1+\delta)$.

The weighted Sobolev spaces $W^{1, p}(\Omega ; \nu)$ and $W_{0}^{1, p}(\Omega ; \nu)$ inherit all the natural properties of the Sobolev spaces $W^{1, p}(\Omega)$ and $W_{0}^{1, p}(\Omega)$, respectively. Since we shall be using De Giorgi type techniques in this paper, we are especially interested in cut-off functions with respect to a level: for $u \in W^{1, p}(\Omega ; \nu)$ and $\lambda \in \mathbb{R}$ the cut-off functions

$$
(u-\lambda)_{+}=\max (u, \lambda)-\lambda \text { and }(u-\lambda)_{-}=\lambda-\min (u, \lambda)
$$

also belong to $W^{1, p}(\Omega ; \nu)$ and their gradients are

$$
\nabla(u-\lambda)_{+}=\left\{\begin{array}{ll}
0 & \text { on }\{u \leq \lambda\}, \\
\nabla u & \text { on }\{u>\lambda\},
\end{array} \quad \nabla(u-\lambda)_{-}= \begin{cases}0 & \text { on }\{u \geq \lambda\}, \\
-\nabla u & \text { on }\{u<\lambda\}\end{cases}\right.
$$

Moreover, if $u \in W_{\text {loc }}^{1, p}(\Omega ; \nu)$ and $t \in \mathbb{R}$, then $\nabla u=0$ almost everywhere on the set $\{u=t\} \cap \Omega$. Next, if $u$ and $v$ are bounded functions in $W^{1, p}(\Omega ; \nu)$, then $u v \in W^{1, p}(\Omega ; \nu)$ and $\nabla(u v)=u \nabla v+v \nabla u$. If, in addition, $v \in W_{0}^{1, p}(\Omega ; \nu)$, then the product satisfies $u v \in W_{0}^{1, p}(\Omega ; \nu)$.

The reader can find more detailed information on $p$-admissible weights in [21].

We need a multiplicative inequality of Sobolev-Gagliardo-Nirenberg type. 
Lemma 4. Let $s \in \mathbb{R}$. For any ball $B$ of radius $R$ in $\mathbb{R}^{n}$ and any function $v \in$ $W_{0}^{1, p}(B ; \nu)$, the following inequality holds:

$$
\frac{1}{\nu(B)} \int_{B}|v|^{p+s h} d \nu \leq C\left(\frac{1}{\nu(B)} \int_{B}|v|^{s} d \nu\right)^{h} \frac{R^{p}}{\nu(B)} \int_{B}|\nabla v|^{p} d \nu,
$$

where $C$ and $h$ are positive constants independent of $B$ and $v$.

Proof. Let $h=1-1 / \kappa$, where $\kappa$ is taken from inequality (1.5). By using this inequality and Hölder's inequality we obtain

$$
\begin{aligned}
\frac{1}{\nu(B)} \int_{B}|v|^{p+s h} d \nu & \leq\left(\frac{1}{\nu(B)} \int_{B}|v|^{s} d \nu\right)^{h}\left(\frac{1}{\nu(B)} \int_{B}|v|^{\kappa p} d \nu\right)^{1 / \kappa} \\
& \leq C_{3}^{p}\left(\frac{1}{\nu(B)} \int_{B}|v|^{s} d \nu\right)^{h} \frac{R^{p}}{\nu(B)} \int_{B}|\nabla v|^{p} d \nu
\end{aligned}
$$

\section{$\S$ 3. An energy estimate}

Lemma 5. Let $u$ be a solution of (1.1) in the cylinder $Q=\Omega \times\left[T_{1}, T_{2}\right]$. Let $\xi$ be a nonnegative Lipschitz function vanishing in a neighbourhood of the lateral boundary of $Q$. Then the following energy estimate holds for any $k \in \mathbb{R}$ :

$$
\begin{aligned}
& \underset{t \in\left(T_{1}, T_{2}\right)}{\operatorname{ess} \sup _{\Omega}} \int_{\Omega}(u-k)_{ \pm}^{2} \xi^{p} d \nu+C_{0} \iint_{Q}|u|^{m-1}\left|\nabla(u-k)_{ \pm}\right|^{p} \xi^{p} d \nu d t \\
& \leq p \iint_{Q}(u-k)_{ \pm}^{2} \xi^{p-1} \xi_{t} d \nu d t \\
& +C_{6} \iint_{Q}|u|^{m-1}(u-k)_{ \pm}^{p}|\nabla \xi|^{p} d \nu d t \\
& +\left.\int_{\Omega}(u-k)_{ \pm}^{2} \xi^{p} d \nu\right|_{t=-T_{1}} \\
& +\iint_{(u-k)_{ \pm}>0}\left(C_{l} \xi+C_{u}(u-k)_{ \pm}|\nabla \xi|\right) \xi^{p-1} d \nu d t
\end{aligned}
$$

where $C_{6}=2\left(p C_{1}\right)^{p}\left(C_{0} / 2\right)^{1-p}$.

Remark. If $u$ is a subsolution of (1.1), then the estimate (3.1) holds for $(u-k)_{+}$, and if $u$ is a supersolution of (1.1), then the estimate (3.1) holds for $(u-k)_{-}$. Thus, the lemmas of the next section based on this inequality are valid for subsolutions in assertions involving $\mu_{+}$and for supersolutions in assertions involving $\mu_{-}$.

\section{$\S$ 4. Auxiliary lemmas}

The following lemma is a central tool in our proof.

Lemma 6. Let $u$ be a nonnegative solution of equation (1.1) in a cylinder $Q=\left(x_{0}, t_{0}\right)+$ $Q(R, T)$. Suppose that $\mu_{+} \geq \operatorname{ess} \sup \{u ; Q\}, \mu_{-} \leq \operatorname{ess} \inf \{u ; Q\}, \omega>0, a \in(0,1)$. Let $Q_{1}=\left(x_{0}, t_{0}\right)+Q(R / 2, T / 2)$. Then there exists a positive constant $\gamma=\gamma($ data $)$ such that, setting

$$
f(\theta, a, \Gamma)=\gamma(1-a)^{(2+p / h)(1+l)} \theta^{-1}\left(1+\Gamma+\frac{1}{\theta}\right)^{-1-1 / h},
$$

the following hold: 
1) if

$$
\nu\left(\left\{u<\mu_{-}+\omega\right\} \cap Q\right)<f(\theta, a, \Gamma) \nu(Q),
$$

where $\theta=\theta\left(T, \mu_{-}, \omega, R\right)$ and $\Gamma=\Gamma\left(\mu_{-}, \omega, R\right)$, then $u \geq \mu_{-}+$aw almost everywhere in the cylinder $Q_{1}$;

2) if

$$
\nu\left(\left\{u>\mu_{+}-\omega\right\} \cap Q\right)<f(\theta, a, \Gamma) \nu(Q),
$$

where $\theta=\theta\left(T, \mu_{+}, \omega, R\right)$ and $\Gamma=\Gamma\left(\mu_{+}, \omega, R\right)$, then $u \leq \mu_{+}-$aw almost everywhere in the cylinder $Q_{1}$.

Proof. We prove both cases simultaneously. For $j=0,1, \ldots$ we introduce the sequence of levels

$$
k_{j}=\mu_{ \pm} \mp\left(a \omega+(1-a) 2^{-j} \omega\right),
$$

where the presence of $\mu_{-}$corresponds to the first case, and $\mu_{+}$to the second case. For $R_{j}=\left(2^{-1}+2^{-j}\right) R$ and $T_{j}=\left(2^{-1}+2^{-j}\right) T$ we consider the sequence of cylinders $Q_{j}=\left(x_{0}, t_{0}\right)+Q\left(R_{j}, T_{j}\right)$. We set

$$
Y_{j}=\nu\left(\left\{\left(u-k_{j}\right)_{ \pm}>0\right\} \cap Q_{j}\right) .
$$

To prove the first part of the lemma we use the family of energy inequalities (3.1) with $(u-k)_{-}$, and to prove the second part of the lemma we use (3.1) with $(u-k)_{+}$.

Let $\xi_{j}$ be smooth nonnegative functions that are equal to zero near the parabolic boundary of $Q_{j}$, are equal to 1 on the cylinder $Q_{j+1}$, and are such that

$$
|\nabla \xi|+\left|\xi_{t}\right| \leq \frac{C}{2^{j}}, \quad C=C(n) .
$$

In inequality (3.1), we estimate the integrands on the right-hand side as follows:

$$
|u|^{m-1}(u-k)_{ \pm}^{p} \leq 2^{m-1} \max \left(\left|\mu_{ \pm}\right|, \omega\right)^{m-1} \omega^{p}, \quad\left(u-k_{j}\right)_{ \pm}^{2} \leq \omega^{2} .
$$

The third integral on the right-hand side of (3.1) vanishes because of the choice of $\xi$. In the second integrand on the left-hand side of (3.1) we use the equality

$$
|u|^{m-1}\left|\nabla(u-k)_{ \pm}\right|^{p}=\frac{1}{p+m-1}\left|\nabla\left(u^{l+1}-k_{j}^{l+1}\right)_{ \pm}\right|^{p}
$$

Thus, we obtain

$$
\begin{aligned}
& \underset{t \in(0, T)}{\operatorname{ess} \sup } \int_{x_{0}+B_{R}}(u\left.-k_{j}\right)_{ \pm}^{2} \xi_{j}^{p} d \nu+\iint_{Q}\left|\nabla\left(u^{l+1}-k_{j}^{l+1}\right)_{ \pm}\right|^{p} \xi_{j}^{p} d \nu d t \\
& \leq C 2^{p j} Y_{j} R^{-p} \omega^{p}\left(\max \left(\left|\mu_{ \pm}\right|, \omega\right)\right)^{m-1}\left(1+\Gamma+\frac{1}{\theta}\right) .
\end{aligned}
$$

Since $p>2$ and $0 \leq \xi_{j} \leq 1$, we can replace $\xi^{p}$ in the second integrand on the left-hand side by $\xi^{p^{2} / 2}$. Obviously, in the same integrand we can introduce $\xi^{p / 2}$ under the sign $\nabla$ at the expense of increasing the constant $C$ on the right-hand side. As a result we obtain

$$
\begin{aligned}
& \underset{t \in(0, T)}{\operatorname{ess} \sup } \int_{x_{0}+B_{R}}(u\left.-k_{j}\right)_{ \pm}^{2} \xi_{j}^{p} d \nu+\iint_{Q}\left|\nabla\left[\left(u^{l+1}-k_{j}^{l+1}\right)_{ \pm} \xi_{j}^{p / 2}\right]\right|^{p} d \nu d t \\
& \leq C 2^{p j} Y_{j} R^{-p} \omega^{p}\left(\max \left(\left|\mu_{ \pm}\right|, \omega\right)\right)^{m-1}\left(1+\Gamma+\frac{1}{\theta}\right) .
\end{aligned}
$$

In order to apply the parabolic Sobolev inequality (2.4), we need to transform the first integral on the left-hand side into an integral of $\left(u^{l+1}-k_{j}^{l+1}\right)_{ \pm}^{2} \xi^{p}$. It is easy to see that

$$
\left(u^{l+1}-k^{l+1}\right)_{ \pm} \leq C \max \left(\left|\mu_{ \pm}\right|, \omega\right)^{l}(u-k)_{ \pm} .
$$


We set $v_{j}=\left(u^{l+1}-k_{j}^{l+1}\right)_{ \pm} \xi_{j}^{p / 2}$. Then from (4.1) we obtain

$$
\begin{gathered}
\left(\max \left(\left|\mu_{ \pm}\right|, \omega\right)\right)^{-2 l} \underset{t \in(0, T)}{\operatorname{ess} \sup } \int_{x_{0}+B_{R}} v_{j}^{2} d \nu+\iint_{Q}\left|\nabla v_{j}\right|^{p} d \nu d t \\
\leq C 2^{p j} Y_{j} R^{-p} \omega^{p}\left(\max \left(\left|\mu_{ \pm}\right|, \omega\right)\right)^{m-1}\left(1+\Gamma+\frac{1}{\theta}\right) .
\end{gathered}
$$

Applying Lemma 4 with the parameter $s=2$ we obtain

$$
\begin{gathered}
\left|k_{j}^{l+1}-k_{j+1}^{l+1}\right|^{p+2 h} Y_{j+1} \leq \int_{Q_{j}} v_{j}^{p+2 h} d \nu d t \\
\leq C 2^{p(1+h) j} R^{-p h}\left(\nu\left(B_{R}\right)\right)^{-h} Y_{j}^{1+h}\left(1+\Gamma+\frac{1}{\theta}\right)^{1+h} \omega^{p(1+h)}\left(\max \left(\left|\mu_{ \pm}\right|, \omega\right)\right)^{(m-1)(1+h)+2 l h} .
\end{gathered}
$$

To obtain an upper estimate for $Y_{j+1}$, we estimate the difference $\left|k_{j}^{l+1}-k_{j+1}^{l+1}\right|$ from below. We have

$$
\begin{aligned}
\left|k_{j}^{l+1}-k_{j+1}^{l+1}\right| & \geq C\left|k_{j}-k_{j+1}\right|\left(\left|k_{j}\right|^{l}+\left|k_{j+1}\right|^{l}\right) \\
& \geq(1-a) \omega 2^{-j(l+1)} \max \left(\left|\mu_{ \pm} \mp a \omega\right|,(1-a) \omega\right)^{l} \\
& \geq(1-a)^{1+l} \omega 2^{-j(l+1)} \max \left(\left|\mu_{ \pm}\right|, \omega\right)^{l} .
\end{aligned}
$$

In both cases we obtain the following estimate for $Y_{j+1}$ :

$$
\frac{Y_{j+1}}{2^{(2 p(1+h)+l) j} Y_{j}^{1+h}} \leq C(1-a)^{-(p+2 h)(1+l)}(\nu(Q))^{-h} \theta^{h}\left(1+\Gamma+\frac{1}{\theta}\right)^{1+h} .
$$

By the Ladyzhenskaya-Ural'tseva lemma on hypergeometric convergence, $Y_{j} \rightarrow 0$ as $j \rightarrow \infty$ under the condition that

$$
Y_{0} \leq \gamma(1-a)^{(2+p / h)(1+l)} \theta^{-1}\left(1+\Gamma+\frac{1}{\theta}\right)^{-1-1 / h} \nu(Q) .
$$

This is the result stated in the lemma.

By following the proof of the preceding lemma, it is easy to obtain the next result.

Lemma 7. Let $u$ be a solution of (1.1) in the cylinder $Q=\left(x_{0}, t_{0}\right)+Q(R, T)$. Suppose that $\mu_{-} \leq \operatorname{ess} \inf \{u ; Q\}, \mu_{+} \geq \operatorname{ess} \sup \{u ; Q\}, \omega>0, a \in(0,1)$. Then there exists a positive constant $\gamma=\gamma($ data $)$ such that

1) if

$$
u\left(\cdot, t_{0}-T\right) \geq \mu_{-}+\omega \text { almost everywhere on } x_{0}+B_{R}
$$

and

$$
\theta=\theta\left(T, \mu_{-}, \omega, R\right)<\gamma(1-a)^{(2+p / h)(1+l)}(1+\Gamma)^{-1-1 / h}, \quad \Gamma=\Gamma\left(\mu_{-}, \omega, R\right),
$$

then $u \geq \mu_{-}+$aw almost everywhere in the cylinder $\left(x_{0}, t_{0}\right)+Q(R / 2, T)$;

2) if

$$
u\left(\cdot, t_{0}-T\right) \leq \mu_{+}-\omega \text { almost everywhere on } x_{0}+B_{R}
$$

and

$$
\theta=\theta\left(T, \mu_{+}, \omega, R\right)<\gamma(1-a)^{(2+p / h)(1+l)}(1+\Gamma)^{-1-1 / h}, \quad \Gamma=\Gamma\left(\mu_{+}, \omega, R\right),
$$

then $u \leq \mu_{+}-$aw almost everywhere in the cylinder $\left(x_{0}, t_{0}\right)+Q(R / 2, T)$. 
Proof. Let $R_{j}=\left(2^{-1}+2^{-j}\right) R, j=0,1,2, \ldots, T_{j}=T$. All the arguments of Lemma 6 are repeated with two differences: a) the cut-off functions $\xi_{j}$ are now taken to be independent of $t$; that is, $\xi=\xi(x)$ is a smooth positive function taking values in $[0,1]$ with compact support in $x_{0}+B_{R_{j}}$, equal to 1 on $x_{0}+B_{R_{j+1}}$, and satisfying $\left|\nabla \xi_{j}\right| \leq C 2^{j} R^{-1}$; b) the cylinders $Q_{j}$ have the same height as the original cylinder; that is, $Q_{j}=\left(x_{0}, t_{0}\right)+$ $Q\left(R_{j}, T\right)$. Since $\xi_{j}$ is independent of $t$, the first term on the right-hand side of the energy estimate (3.1), which contains the derivative of $\xi_{j}$ with respect to time, vanishes. Consequently, all the estimates that we obtained in the proof of Lemma 6. starting with (4.1), are true. Here, $(1+\Gamma+1 / \theta)$ is replaced by $(1+\Gamma)$. Thus, we arrive at the following conclusion:

1) if

$$
\frac{\nu\left(\left\{u>\mu_{-}+\omega\right\} \cap Q\right)}{\nu(Q)}<\gamma(1-a)^{(2+p / h)(1+l)} \theta^{-1}(1+\Gamma)^{-1-1 / h},
$$

then $u \geq \mu_{-}+a \omega$ almost everywhere in the cylinder $\left(x_{0}, t_{0}\right)+Q(R / 2, T)$;

2) if

$$
\frac{\nu\left(\left\{u<\mu_{+}-\omega\right\} \cap Q\right)}{\nu(Q)}<\gamma(1-a)^{(2+p / h)(1+l)} \theta^{-1}(1+\Gamma)^{-1-1 / h},
$$

then $u \leq \mu_{+}-a \omega$ almost everywhere in the cylinder $\left(x_{0}, t_{0}\right)+Q(R / 2, T)$.

The constant $\gamma=\gamma($ data $)>0$ in (4.3) and (4.4) is the constant in the statement of the lemma. Obviously, the left-hand side of (4.3) and (4.4) is no greater than 1 . On the other hand, if $\theta$ satisfies the hypothesis of the lemma, then the right-hand side of (4.3) (or (4.4) ) is greater than 1. Thus, under the hypotheses of the lemma we have (4.3) (or (4.4)), which fact implies the required assertion.

A direct consequence of this lemma is the following assertion.

Lemma 8. Let $u$ be a solution of (1.1) in the cylinder $Q=\left(x_{0}, t_{0}\right)+Q(2 R, T)$. Suppose that $\mu_{-} \leq \operatorname{ess} \inf \{u, Q\}, \mu_{+} \geq \operatorname{ess} \sup \{u ; Q\}, \omega>0$. Let $\theta_{ \pm}=\theta\left(T, \mu_{ \pm}, \omega, 2 R\right)$. Then there exists a constant $\gamma=\gamma($ data $)>0$ such that

1) if for some $t_{*} \in\left(t_{0}-T, t_{0}\right)$ and $\tau \in(0,1)$ the inequality $u\left(x, t_{*}\right)<\mu_{+}-\tau \omega$ holds almost everywhere on $x_{0}+B_{2 R}$, then $u \leq \mu_{+}-\xi \omega / 2$ almost everywhere in the cylinder $\left(x_{0}, t_{0}\right)+Q\left(R, t_{0}-t_{*}\right)$, where $\xi=\min \left(\tau, \delta_{0}\right), \delta_{0}=\left(\gamma / \theta_{+}\right)^{1 /(p-2)}$, provided that $\Gamma\left(\mu_{+}, \xi \omega, R\right) \leq 1$

2) if for some $t_{*} \in\left(t_{0}-T, t_{0}\right)$ and $\tau \in(0,1)$ the inequality $u\left(x, t_{*}\right)>\mu_{-}+\tau \omega$ holds almost everywhere on $x_{0}+B_{2 R}$, then $u \geq \mu_{-}+\xi \omega / 2$ almost everywhere in the cylinder $\left(x_{0}, t_{0}\right)+Q\left(R, t_{0}-t_{*}\right)$, where $\xi=\min \left(\tau, \delta_{0}\right), \delta_{0}=\left(\gamma / \theta_{-}\right)^{1 /(p-2)}$, provided that $\Gamma\left(\mu_{-}, \xi \omega, R\right) \leq 1$.

Proof. We set

$$
Q_{1}=\left(x_{0}, t_{0}\right)+Q\left(R, t_{0}-t_{*}\right) \text { and } Q_{2}=\left(x_{0}, t_{0}\right)+Q\left(2 R, t_{0}-t_{*}\right) .
$$

We will prove the first part of the lemma. Obviously, $u\left(x, t_{*}\right) \leq \mu_{+}-\xi \omega$ almost everywhere on the set $x_{0}+B_{2 R}$ for any $\xi \in[0, \tau]$. Applying Lemma 7 with $a=1 / 2$ we have $u \leq \mu_{+}-\xi \omega / 2$ almost everywhere in the cylinder $Q_{1}$ if

$$
\frac{T}{\max \left(\left|\mu_{+}\right|, \xi \omega\right)^{1-m}(\xi \omega)^{2-p}(2 R)^{p}} \leq \gamma,
$$

where $\gamma=\gamma($ data $)>0$. Since the left-hand side of (4.5) does not exceed $\xi^{p-2} \theta_{+}$, we can take $\xi=\min \left(\tau,\left(\gamma / \theta_{+}\right)^{1 /(p-2)}\right)$, which gives the required estimate. The proof of the second case is similar. 
Remark. The estimate in Lemma 8 is fairly crude. For example, in the case $\mu=0$ the correct exponent in the definition of $\delta_{0}$ is $\frac{1}{m+p-3}$. But the form given above is sufficient for our purposes.

Lemma 9. Let $u$ be a solution of (1.1) in a cylinder $Q=\left(x_{0}, t_{0}\right)+Q(R, T)$. Suppose that $\mu_{-} \leq \operatorname{ess} \inf \{u ; Q\}, \mu_{+} \geq \operatorname{ess} \sup \{u ; Q\}, \omega>0, \gamma \in(0,1)$. Then there exist positive constants $\theta_{*}, \delta, \sigma \in(0,1)$ depending only on the parameters of the equation and $\gamma$ such that

1) if

$\nu\left(\left\{u\left(\cdot, t_{0}-T\right)>\mu_{-}+\omega\right\} \cap\left(x_{0}+B_{R}\right)\right) \geq \gamma \nu\left(x_{0}+B_{R}\right) \quad$ and $\quad \theta=\theta\left(T, \mu_{-}, \omega, R\right)<\theta_{*}$,

then

$$
\nu\left(\left\{u(\cdot, t)>\mu_{-}+(1-\delta) \omega\right\} \cap\left(x_{0}+B_{R}\right)\right) \geq \frac{\gamma}{2} \nu\left(x_{0}+B_{R}\right)
$$

for all $t \in\left(t_{0}-T, t_{0}\right)$, provided that $\Gamma\left(\mu_{-}, \omega, \sigma R\right) \leq 1$;

2) if

$\nu\left(\left\{u\left(\cdot, t_{0}-T\right)<\mu_{+}-\omega\right\} \cap\left(x_{0}+B_{R}\right)\right) \geq \gamma \nu\left(x_{0}+B_{R}\right) \quad$ and $\quad \theta=\theta\left(T, \mu_{+}, \omega, R\right)<\theta_{*}$,

then

$$
\nu\left(\left\{u(\cdot, t)<\mu_{+}-(1-\delta) \omega\right\} \cap\left(x_{0}+B_{R}\right)\right) \geq \frac{\gamma}{2} \nu\left(x_{0}+B_{R}\right)
$$

for all $t \in\left(t_{0}-T, t_{0}\right)$, provided that $\Gamma\left(\mu_{+}, \omega, \sigma R\right) \leq 1$.

Proof. The proof is the same for both cases; therefore we only prove the first case. Let $k=\mu_{-}+\omega$. Let $\sigma \in(0,1)$ and $\delta>0$. Let $\xi=\xi(x)$ be a smooth nonnegative function with values in $[0,1]$ that vanishes in a neighbourhood of the boundary of $x_{0}+B_{R}$, is equal to 1 on $x_{0}+B_{(1-\sigma) R}$, and is such that $|\nabla \xi| \leq C /(\sigma R)$ for $C=C(n)$. From the energy estimate (3.1) we obtain

$$
\begin{gathered}
(\delta \omega)^{2} \nu\left(\left\{u(x, t) \leq \mu_{-}+(1-\delta) \omega\right\} \cap\left(x_{0}+B_{(1-\sigma) R}\right)\right) \leq \int_{x_{0}+B_{R}}(u-k)_{-}^{2}(\cdot, t) \xi^{p} d \nu \\
\leq \int_{x_{0}+B_{R}}(u-k)_{-}^{2}\left(\cdot, t_{0}-T\right) \xi^{p} d \nu+C \int_{Q}|u|^{m-1}(u-k)_{-}^{p}|\nabla \xi|^{p} d \nu d t+\left(C_{l}+\frac{C_{u} \omega}{\sigma R}\right) \nu(Q) \\
\leq\left[(1-\gamma) \omega^{2}+C \theta \omega^{2} \sigma^{-p}+\Gamma\left(\mu_{-}, \omega, \sigma R\right) \theta \omega^{2} \sigma^{-p}\right] \nu\left(x_{0}+B_{R}\right) .
\end{gathered}
$$

Hence for all $t \in\left(t_{0}, t_{0}-T\right)$ we have

$$
\begin{aligned}
& \frac{\nu\left(\left\{u(x, t) \leq \mu_{-}+(1-\delta) \omega\right\} \cap\left(x_{0}+B_{R}\right)\right)}{\nu\left(x_{0}+B_{R}\right)} \\
& \leq \frac{1-\gamma}{\delta^{2}}+\frac{\left(C+\Gamma\left(\mu_{-}, \omega, \sigma R\right)\right) \theta \sigma^{-p}}{\delta^{2}}+\frac{\nu\left(x_{0}+B_{R} \backslash B_{(1-\sigma) R}\right)}{\nu\left(x_{0}+B_{R}\right)} .
\end{aligned}
$$

First we choose $\delta$ in such a way that $(1-\gamma) / \delta^{2}=1-3 \gamma / 4$. Next, we choose $\sigma$ in such a way that $\nu\left(x_{0}+B_{R} \backslash B_{(1-\sigma) R}\right) \leq \gamma \nu\left(x_{0}+B_{R}\right) / 8$. To complete the proof it remains to choose $\theta$ in such a way that $\left(C+\Gamma\left(\mu_{-}, \omega, \sigma R\right)\right) \theta \sigma^{-p} / \delta^{2} \leq \gamma / 8$.

The following lemma is known by the name 'telescopic argument'.

Lemma 10. Let $u$ be a solution of (1.1) in a cylinder $Q=\left(x_{0}, t_{0}\right)+Q(2 R, 2 T)$, let $\mu_{-} \leq \operatorname{ess} \inf \{u ; Q\}, \mu_{+} \geq \operatorname{ess} \sup \{u ; Q\}, \omega>0$. We set $Q_{1}=\left(x_{0}, t_{0}\right)+Q(R, T)$.

1. Suppose that

$$
\nu\left(\left\{u(\cdot, t)>\mu_{-}+\omega\right\} \cap\left(x_{0}+B_{R}\right)\right)>\gamma \nu\left(x_{0}+B_{R}\right), \quad \gamma>0,
$$

for all $t \in\left(t_{0}-T, t_{0}\right)$. Then for any $\varepsilon>0$ there exists $\delta=\delta(\varepsilon, \gamma$, data $)>0$ such that if $\theta\left(T, \mu_{-}, \delta \omega, R\right) \geq 1$ and $\Gamma\left(\mu_{-}, \delta \omega, R\right) \leq 1$, then

$$
\nu\left(\left\{u \leq \mu_{-}+\delta \omega\right\} \cap Q_{1}\right)<\varepsilon \nu\left(Q_{1}\right) .
$$




\section{Suppose that}

$$
\nu\left(\left\{u(\cdot, t)<\mu_{+}-\omega\right\} \cap\left(x_{0}+B_{R}\right)\right)>\gamma \nu\left(x_{0}+B_{R}\right), \quad \gamma>0,
$$

for all $t \in\left(t_{0}-T, t_{0}\right)$. Then for any $\varepsilon>0$ there exists $\delta=\delta(\varepsilon, \gamma$, data $)>0$ such that if $\theta\left(T, \mu_{+}, \delta \omega, R\right) \geq 1$ and $\Gamma\left(\mu_{+}, \delta \omega, R\right) \leq 1$, then

$$
\nu\left(\left\{u \geq \mu_{+}-\delta \omega\right\} \cap Q_{1}\right)<\varepsilon \nu\left(Q_{1}\right) .
$$

Proof. We take a smooth cut-off function $\xi$ that vanishes near the parabolic boundary of $Q$, is equal to 1 on $Q_{1}$, takes values in $[0,1]$, and is such that $|\nabla \xi| \leq 4 / R,\left|\xi_{t}\right| \leq 4 / T$. Let $k_{j}=\mu_{-}+2^{-j} \omega$ for $j=0,1, \ldots, j_{*}$. Repeating the arguments given above we obtain

$$
\int_{Q_{1}}\left|\nabla\left(u^{l+1}-k_{j}^{l+1}\right)_{-}\right|^{p} d \nu d t \leq C 2^{-p j} \omega^{p} \max \left(\left|\mu_{-}\right|, 2^{-j} \omega\right)^{m-1} R^{-p} \nu(Q)
$$

under the condition that $\theta\left(T, \mu_{-}, 2^{-j} \omega, R\right) \geq 1 / 2$ and $\Gamma\left(\mu_{-}, 2^{-j} \omega, R\right) \leq 1$. We put

$$
\begin{gathered}
B=x_{0}+B_{R}, \quad A_{j}=\left\{u \leq k_{j}\right\} \cap Q_{1}, \quad \bar{A}_{j}=\left\{u>k_{j}\right\} \cap Q_{1}, \\
A_{j}(t)=\left\{u(\cdot, t) \leq k_{j}\right\} \cap B, \quad \bar{A}_{j}(t)=\left\{u(\cdot, t)>k_{j}\right\} \cap B .
\end{gathered}
$$

Next, we need a consequence of Poincaré's inequality, which plays the same role as the De Giorgi-Poincaré inequality in the nonweighted case. Let $\varphi$ be a nonnegative function in $W^{1, p}(B, \nu)$. Obviously,

$$
\bar{\varphi}=\frac{1}{\nu(B)} \int_{B} \varphi d \nu \geq k \frac{\nu(\{\varphi \geq k\} \cap B)}{\nu(B)} .
$$

On the other hand, for any $\beta>0$ we have

$$
\begin{aligned}
\frac{1}{\nu(B)} \int_{B}|\varphi-\bar{\varphi}|^{\beta} d \nu & \geq \frac{\nu(\{\varphi=0\} \cap B)}{\nu(B)} \bar{\varphi}^{\beta} \\
& \geq\left(k \frac{\nu(\{\varphi \geq k\} \cap B)}{\nu(B)}\right)^{\beta} \frac{\nu(\{\varphi=0\} \cap B)}{\nu(B)} .
\end{aligned}
$$

Since any $p$-admissible weight is $q$-admissible for some positive $q<p$ determined by the constants involved in the conditions of $p$-admissibility (see [22]), we can use Poincaré's $(q, q)$-inequality with $\varphi=\left(u^{l+1}-k_{j}^{l+1}\right)$ - to obtain

$$
\begin{aligned}
\gamma\left(\mid k_{j}^{l+1}\right. & \left.-k_{j+1}^{l+1} \mid \frac{\nu\left(A_{j+1}(t)\right)}{\nu(B)}\right)^{q} \leq\left(\left|k_{j}^{l+1}-k_{j+1}^{l+1}\right| \frac{\nu\left(A_{j+1}(t)\right)}{\nu(B)}\right)^{q} \frac{\nu\left(\bar{A}_{j}(t)\right)}{\nu(B)} \\
& \leq C \frac{R^{q}}{\nu(B)} \int_{A_{j}(t) \backslash A_{j+1}(t)}\left|\nabla\left(u^{l+1}-k_{j}^{l+1}\right)_{-}\right|^{q} d \nu \\
& \leq C \frac{R^{q}}{\nu(B)}\left(\nu\left(A_{j}(t) \backslash A_{j+1}(t)\right)\right)^{1-q / p}\left(\int_{A_{j}(t) \backslash A_{j+1}(t)}\left|\nabla\left(u^{l+1}-k_{j}^{l+1}\right)_{-}\right|^{p} d \nu\right)^{q / p} .
\end{aligned}
$$

Hence,

$$
\begin{aligned}
& \left|k_{j}^{l+1}-k_{j+1}^{l+1}\right| \frac{\nu\left(A_{j+1}(t)\right)}{\nu(B)} \\
& \quad \leq C R\left(\frac{1}{\nu(B)} \int_{A_{j}(t) \backslash A_{j+1}(t)}\left|\nabla\left(u^{l+1}-k_{j}^{l+1}\right)_{-}\right|^{p} d \nu\right)^{1 / p}\left(\frac{\nu\left(A_{j}(t) \backslash A_{j+1}(t)\right)}{\nu(B)}\right)^{1 / q-1 / p} .
\end{aligned}
$$

Integrating this inequality with respect to $t \in\left[t_{0}-T, t_{0}\right]$ and applying the triple Hölder inequality with exponents $(1 / p)^{-1},(1 / q-1 / p)^{-1}$, and $(1-1 / q)^{-1}$ to the right-hand side, 
we obtain

$$
\begin{aligned}
& \left|k_{j}^{l+1}-k_{j+1}^{l+1}\right| \frac{\nu\left(A_{j+1}\right)}{\nu(B)} \\
& \quad \leq C R\left(\frac{1}{\nu(B)} \int_{A_{j} \backslash A_{j+1}}\left|\nabla\left(u^{l+1}-k_{j}^{l+1}\right)_{-}\right|^{p} d \nu d t\right)^{1 / p}\left(\frac{\nu\left(A_{j} \backslash A_{j+1}\right)}{\nu(B)}\right)^{1 / q-1 / p} T^{1-1 / q} .
\end{aligned}
$$

It is easy to see that

$$
\left|k_{j}^{l+1}-k_{j+1}^{l+1}\right| \geq C \max \left(\left|\mu_{-}\right|, 2^{-j} \omega\right)^{l} 2^{-j-1} \omega .
$$

By using this estimate in the preceding inequality we arrive at

$$
\frac{\nu\left(A_{j+1}\right)}{\nu\left(Q_{1}\right)} \leq C\left(\frac{\nu\left(A_{j} \backslash A_{j+1}\right)}{\nu\left(Q_{1}\right)}\right)^{1 / q-1 / p} .
$$

Raising this inequality to the power $(1 / q-1 / p)^{-1}$, summing with respect to $j$, and using the fact that the sequence $\nu\left(A_{j}\right)$ is nonincreasing, for any $j$ we obtain

$$
(j+1)\left(\frac{\nu\left(A_{j+1}\right)}{\nu\left(Q_{1}\right)}\right)^{(1 / q-1 / p)^{-1}} \leq \sum_{i=0}^{j}\left(\frac{\nu\left(A_{i+1}\right)}{\nu\left(Q_{1}\right)}\right)^{(1 / q-1 / p)^{-1}} \leq C \sum_{i=0}^{j} \frac{\nu\left(A_{i} \backslash A_{i+1}\right)}{\nu\left(Q_{1}\right)} \leq C .
$$

Thus, for any $j \geq 1$ we have

$$
\frac{\nu\left(A_{j}\right)}{\nu\left(Q_{1}\right)} \leq\left(\frac{C}{j}\right)^{1 / q-1 / p}
$$

In order to complete the proof of the lemma in this case, it remains to choose $j_{*}$ such that $C / j_{*} \leq \varepsilon^{p q /(p-q)}$. Then $\delta=2^{-j_{*}}$. Since $\theta\left(T, \mu_{-}, 2^{-j} \omega, R\right)$ is decreasing with respect to $j$, while $\Gamma\left(\mu_{-}, 2^{-j} \omega, R\right)$ is increasing, it is sufficient to require that the conditions $\theta\left(T, \mu_{-}, 2^{-j} \omega, R\right) \geq 1$ and $\Gamma\left(\mu_{-}, 2^{-j} \omega, R\right) \leq 1$ hold at the last step. The proof for the second case is quite similar.

Remark. The constants in the technical lemmas of this section are stable as $p \rightarrow 2$, $m \rightarrow 1$. To stabilise the constants of Hölder continuity as $p \rightarrow 2, m \rightarrow 1$ we need a modification of Lemma 10. The following should be added to the statement. If $\delta \omega<|\mu|$, then there exists $\sigma=\sigma(\varepsilon, \gamma$, data $)>0$ such that the condition $\theta(T, \mu, \delta \omega, R) \geq 1$ can be replaced by $\theta(T, \mu, \omega, R) \geq 1$ if $p<2+\sigma$. The quantity $\sigma$ is independent of $p$. If $\delta \omega>|\mu|$, then we can perform such a change under the condition that $m+p<3+\sigma$, where $\sigma$ is independent of $m, p$. To do this we need to observe that for $\sigma=j_{*}^{-1}$ the aforementioned conditions guarantee that $\theta\left(T, \mu, 2^{-j} \omega, R\right) \geq 1 / 2$ for $j=1, \ldots, j_{*}$ if $\theta(T, \mu, \omega, R) \geq 1$. Next, the condition $\theta(T, \mu, \omega, R)>1$ can be replaced by $\theta(T, \mu, \omega, R)>\lambda$ for any fixed $\lambda>0$. Here, the quantity $C$ in inequality (4.7) will be replaced by $C\left(1+\lambda^{-1}\right)$. In accordance with this, the quantities $\delta, \sigma$ will also depend on $\lambda$.

\section{$\S$ 5. Proof of Hölder Continuity}

Lemma 11. Let $u$ be a solution of (1.1) in a cylinder $Q=\left(x_{0}, t_{0}\right)+Q(8 R, T)$. Put $T_{+}=T_{+}(u, Q), Q_{\text {top }}=\left(x_{0}, t_{0}\right)+Q\left(R, T_{+}\right), \mu_{-}=\operatorname{ess} \inf \{u ; Q\}, \mu_{+}=\operatorname{ess} \sup \{u ; Q\}$ and $\omega=\left(\mu_{+}-\mu_{-}\right) / 2$. Then there exist $N=N($ data $) \in \mathbb{N}, \delta=\delta$ (data) $>0$, and $C=C$ (data) $>0$ such that if $T \geq 2 N T_{+}(u, Q)$ and $\Gamma\left(\mu_{+}, \omega, R\right) \leq C$, then

$$
\operatorname{ess} \operatorname{osc}\left\{u ; Q_{\text {top }}\right\}<(1-\delta) \operatorname{ess} \operatorname{osc}\{u ; Q\} \text {. }
$$

Proof. We write $T_{ \pm}=T_{ \pm}(u, Q)$. Note that

$$
4^{1-m} \leq\left(\frac{\max \left(\left|\mu_{+}\right|,\left|\mu_{+}-\mu_{-}\right| / 2\right)}{\max \left(\left|\mu_{-}\right|,\left|\mu_{+}-\mu_{-}\right| / 2\right)}\right)^{m-1} \leq 4^{m-1}
$$


by the triangle inequality. Therefore,

$$
4^{1-m} \leq \frac{T_{-}}{T_{+}} \leq 4^{m-1}, \quad 4^{1-m} \leq \frac{\Gamma\left(\mu_{-}, \omega, R\right)}{\Gamma\left(\mu_{+}, \omega, R\right)} \leq 4^{m-1} .
$$

Let $T \geq 2 N T_{+}$, where $N \geq 2$ is a positive integer, which will be chosen later. For $j=0, \ldots, N$ we set $t_{j}=t_{0}-j T_{0}$. Consider the cylinders $Q_{j}=\left(x_{0}, t_{j}\right)+Q\left(4 R, T_{+}\right)$, $Q^{(1)}=\left(x_{0}, t_{0}\right)+Q\left(4 R, N T_{+}\right)$and $Q^{(2)}=\left(x_{0}, t_{0}\right)+Q\left(2 R, N T_{+} / 2\right)$.

We apply Lemma [6] with $a=1 / 2$ in the cylinders $Q_{j}, j=1, \ldots, N$. This lemma asserts that if

$$
\nu\left(\left\{u>\mu_{+}-\omega\right\} \cap Q_{j}\right)<\gamma \theta^{-1}\left(1+\frac{1}{\theta}\right)^{-1-1 / h} \nu\left(Q_{j}\right),
$$

where $\gamma=\gamma($ data $)>0, \theta=\theta\left(T_{+}, \mu_{+}, \omega, 4 R\right)$, and $\Gamma\left(\mu_{+}, \omega, 4 R\right) \leq 1$, then $u \leq \mu_{+}-\omega / 2$ in the cylinder $\left(x_{0}, t_{j}\right)+Q\left(2 R, T_{+} / 2\right)$. It is easy to see that $\theta=2^{p}$. Thus, there exists a small positive number $\varepsilon_{0}=\varepsilon_{0}$ (data) such that if in some $Q_{j}$ we have

$$
\nu\left(\left\{u>\mu_{+}-\omega\right\} \cap Q_{j}\right)<\varepsilon_{0} \nu\left(Q_{j}\right),
$$

then

$$
u \leq \mu_{+}-\frac{\omega}{2} \text { in the cylinder }\left(x_{0}, t_{j}\right)+Q\left(2 R, \frac{T_{+}}{2}\right) .
$$

By Lemma 8 applied in the cylinder $\left(x_{0}, t_{0}\right)+Q\left(2 R, t_{0}-t_{j}\right)$, the last estimate implies that

$$
\text { ess } \sup \left\{u ; Q_{\text {top }}\right\} \leq \mu_{+}-\gamma N^{1 /(2-p)} \omega
$$

if $\Gamma\left(\mu_{+}, \xi \omega, R\right) \leq 1$, where $\xi=\min \left(1 / 2,(\gamma / N)^{1 /(p-2)}\right)$. This gives the required decrease in oscillation once we fix the number $N$.

We now pass to the second part of the alternative: when

$$
\nu\left(\left\{u>\mu_{+}-\omega\right\} \cap Q_{j}\right)>\varepsilon_{0} \nu\left(Q_{j}\right)
$$

in all the cylinders $Q_{j}, j=1, \ldots, N$.

This implies that for all $j$ there exists a moment of time $\tau_{j} \in\left(t_{j+1}, t_{j}\right)$ such that

$$
\nu\left(\left\{u\left(\cdot, \tau_{j}\right)>\mu_{-}+\omega\right\} \cap\left(x_{0}+B_{4 R}\right)\right)>\varepsilon_{0} \nu\left(x_{0}+B_{4 R}\right) .
$$

Using Lemma 9 we can extend this estimate of measure forward in time up to time $t_{j-1}$. Let $Q_{j}^{(1)}=\left(x_{0}, t_{j-1}\right)+Q\left(4 R, t_{j-1}-\tau_{j}\right)$ and $\xi \in(0,1)$. Obviously, we can write $\xi \omega$ instead of $\omega$ in (5.1). Lemma 9 asserts that there exists a number $\delta_{1}=\delta_{1}$ (data) $>0$ such that

$$
\nu\left(\left\{u(\cdot, t)>\mu_{-}+\delta_{1} \xi \omega\right\} \cap\left(x_{0}+B_{4 R}\right)\right)>\frac{\varepsilon_{0}}{2} \nu\left(x_{0}+B_{4 R}\right)
$$

for all $t \in\left(\tau_{j}, t_{j-1}\right)$ if only

$$
\theta\left(t_{j-1}-\tau_{j}, \mu_{-}, \xi \omega, 4 R\right)<\theta_{*}, \quad \text { where } \theta_{*}=\theta_{*}(\text { data })>0,
$$

and

$$
\Gamma\left(\mu_{-}, \xi \omega, 4 \sigma R\right) \leq C, \quad \text { where } C=C(\text { data }), \quad \sigma=\sigma(\text { data }) \in(0,1)
$$

Since

$$
\theta\left(t_{j-1}-\tau_{j}, \mu_{-}, \xi \omega, 4 R\right) \leq \xi^{p-2} 2^{p} \frac{T_{+}}{T_{-}} \leq C(m, p) \xi^{p-2},
$$

inequality (5.2) holds with $\xi=\xi$ (data) $>0$ for all $j$. Thus, for all $t \in\left(t_{N}, t_{0}\right)$ we have

$$
\nu\left(\left\{u(\cdot, t)>\mu_{-}+\alpha \omega\right\} \cap\left(x_{0}+B_{4 R}\right)\right)>\frac{\varepsilon_{0}}{2} \nu\left(x_{0}+B_{4 R}\right), \quad \text { where } \alpha=\alpha(\text { data })>0,
$$


under the condition that $\Gamma\left(\mu_{-}, \xi \omega, 4 \sigma R\right) \leq 1$. By Lemma 10 for any $\varepsilon>0$ there exists $\delta=\delta(\varepsilon)>0$ such that if

$$
\theta_{2}(\varepsilon)=\theta\left(N T_{+}, \mu_{-}, \alpha \delta(\varepsilon) \omega, 4 R\right) \geq 1 \quad \text { and } \quad \Gamma\left(\mu_{-}, \alpha \delta(\varepsilon) \omega, 4 R\right) \leq 1,
$$

then

$$
\nu\left(\left\{u<\mu_{-}+\alpha \delta(\varepsilon) \omega\right\} \cap Q^{(1)}\right)<\varepsilon \nu\left(Q^{(1)}\right) .
$$

We set

$$
\theta_{3}(\varepsilon)=\theta\left(T_{+}, \mu_{-}, \alpha \delta(\varepsilon) \omega, 4 R\right)
$$

It is easy to see that

$$
\theta_{2}(\varepsilon)=N \theta_{3}(\varepsilon)=N\left(\frac{\max \left(\left|\mu_{-}\right|, \alpha \delta(\varepsilon) \omega\right)}{\max \left(\left|\mu_{+}\right|, \omega\right)}\right)^{m-1}(\alpha \delta(\varepsilon))^{p-2} 2^{p} \geq C N(\alpha \delta(\varepsilon))^{m+p-3} .
$$

Thus, if we choose an integer $N \in\left[1 / \theta_{3}(\varepsilon), 1 / \theta_{3}(\varepsilon)+1\right)$, then (5.3) holds, and therefore (5.4) also holds. For this choice of $N=N(\varepsilon)$ we have

$$
1 \leq \theta_{2}(\varepsilon) \leq 1+\theta_{3}(\varepsilon) \leq C(m, p) .
$$

We now apply Lemma 6 with $a=1 / 2$ in the cylinder $Q^{(1)}$. This lemma asserts that if

$$
\nu\left(\left\{u<\mu_{-}+\alpha \delta(\varepsilon) \omega\right\} \cap Q^{(1)}\right)<H\left(\theta_{2}(\varepsilon)\right),
$$

where $H(\theta)=C \theta^{-1}(1+1 / \theta)^{-1-1 / h}, C=C($ data $)>0$, and $\Gamma\left(\mu_{-}, \alpha \delta(\varepsilon), 4 R\right) \leq 1$, then

$$
u \geq \mu_{-}+\alpha \delta(\varepsilon) \frac{\omega}{2} \quad \text { almost everywhere in the cylinder } Q^{(2)} .
$$

Since $\theta_{2}(\varepsilon) \in[1, C(m, p)]$ holds for our choice of $N=N(\varepsilon)$, it follows that for all $\varepsilon \in(0,1)$ we have $H\left(\theta_{2}(\varepsilon)\right) \in\left(\gamma_{3}, \gamma_{4}\right)$, where $\gamma_{3,4}=\gamma_{3,4}$ (data) $>0$. We take $\varepsilon=\gamma_{3}$. For this choice of $\varepsilon$ we find values of $N=N(\varepsilon)$ and $\delta=\delta(\varepsilon)$. For this choice, (5.5) holds, and therefore (5.6) also holds, which yields the required decrease in oscillation provided that

$$
\Gamma\left(\mu_{+}, \gamma N^{1 /(2-p)} \omega, 4 R\right) \leq 1, \quad \Gamma\left(\mu_{-}, \xi \omega, 4 \sigma R\right) \leq 1, \quad \Gamma\left(\mu_{-}, \alpha \delta \omega, 4 R\right) \leq 1,
$$

where $\xi, \gamma, \sigma$ and $\alpha$ are positive constants depending only on the data of the problem. It is easy to verify that there exists a constant $C=C$ (data) $>0$ such that these conditions hold if $\Gamma\left(\mu_{+}, \omega, R\right) \leq C$.

Remark. The estimates obtained in the proof of Lemma 11 degenerate as $p \rightarrow 2$. We will show that the constants in this lemma are stable as $p \rightarrow 2$. We will not write out the conditions on $\Gamma(\ldots)$; in the end they also reduce to $\Gamma\left(\mu_{+}, \omega, R\right) \leq C=C$ (data).

We will assume without loss of generality that $\left|\mu_{-}\right|>\omega$. Let $\lambda=\lambda$ (data) be a sufficiently small number. This number will be fixed in the course of the proof. The following alternative holds. In the cylinder

$$
Q_{3}=\left(x_{0}+B_{4 R}\right) \times\left[t_{0}-2 \lambda T_{+}, t_{0}-\lambda T_{+}\right]
$$

we have either

$$
\nu\left(\left\{u>\mu_{+}-\omega\right\} \cap Q_{3}\right)<\varepsilon_{0} \nu\left(Q_{3}\right)
$$

or

$$
\nu\left(\left\{u>\mu_{-}+\omega\right\} \cap Q_{3}\right) \geq \varepsilon_{0} \nu\left(Q_{3}\right) .
$$

We choose the number $\varepsilon_{0}=\varepsilon_{0}(\lambda)$ in such a way that we can apply Lemma 6 in $Q_{3}$ with the parameter $a=1 / 2$. Thus, if the first alternative (5.7) holds, we obtain $u\left(x, t_{0}-\right.$ $\left.\lambda T_{+}\right) \leq \mu_{+}-\omega / 2,\left|x-x_{0}\right|<2 R$. Next, we use Lemma 7 with the parameters $a=1 / 2$ and $\omega / 2$ instead of $\omega$. We obtain $u \leq \mu_{+}-\omega / 4$ in the cylinder $\left(x_{0}, t_{0}\right)+Q\left(R, \lambda T_{+}\right)$ 
under the condition that $\lambda \leq \lambda_{1}$ (data). Now suppose that (5.8) holds. Then there exists $t_{*} \in\left[t_{0}-2 \lambda T_{+}, t_{0}-\lambda T_{+}\right]$such that

$$
\nu\left(\left\{u\left(\cdot, t_{*}\right)>\mu_{-}+\omega\right\} \cap\left(x_{0}+B_{4 R}\right)\right) \geq \varepsilon_{0} \nu\left(x_{0}+B_{4 R}\right) .
$$

Applying Lemma 9, we see that $\delta_{1}$ exists such that

$$
\nu\left(\left\{u>\mu_{-}+\delta_{1} \omega\right\} \cap\left(x_{0}+B_{4 R}\right)\right) \geq \frac{\varepsilon_{0}}{2} \nu\left(x_{0}+B_{4 R}\right), \quad t_{*} \leq t \leq t_{0},
$$

provided that $\lambda \leq \lambda_{2}$ (data). We choose $\lambda=\min \left(\lambda_{1}, \lambda_{2}\right)$ and fix $\varepsilon_{0}$ and $\delta_{1}$ accordingly. In the cylinder $Q_{4}=\left(x_{0}, t_{0}\right)+Q\left(8 R, \theta_{*} T_{+}\right)$, we now apply Lemma 10, taking account of the remark. We find that for any $\varepsilon$ there exist $\delta(\varepsilon), \sigma(\varepsilon)>0$ such that for $Q_{5}=$ $\left(x_{0}, t_{0}\right)+Q\left(4 R, \theta_{*} T_{+} / 2\right)$ we have

$$
\nu\left(\left\{u<\mu_{-}+\delta_{1} \delta(\varepsilon) \omega\right\} \cap Q_{5}\right)<\varepsilon \nu\left(Q_{5}\right), \quad \delta(\varepsilon)^{2-p} \leq 2,
$$

under the condition that $p<2+\sigma(\varepsilon)$. It remains to take $\varepsilon$ such that we can apply Lemma [6 in $Q_{5}$ with the parameter $a=1 / 2$ and with $\delta_{1} \delta \omega$ instead of $\omega$. It is easy to see that

$$
\theta+\theta^{-1} \leq C \text { (data) }, \quad \theta=\theta\left(\frac{\lambda T_{+}}{2}, \mu_{-}, \delta_{1} \delta \omega, 4 R\right) .
$$

Therefore we can choose $\varepsilon=\varepsilon$ (data) such that

$$
u \geq \mu_{-}+\frac{1}{2} \delta_{1} \delta(\varepsilon) \omega \quad \text { in the cylinder }\left(x_{0}, t_{0}\right)+Q\left(2 R, \frac{\lambda T_{+}}{4}\right)
$$

if $p<2+\sigma(\varepsilon)$. All the constants in this proof can be considered to be independent of $p$ if $p$ is close to 2 .

Thus, we find numbers $\theta_{*}=\theta_{*}($ data $)>0, \sigma=\sigma($ data $)>0, \gamma=\gamma($ data $)>0$ independent of $p$ such that if the solution $u$ is defined in the cylinder $\mathcal{Q}=\left(x_{0}, t_{0}\right)+$ $Q(8 R, T)$, where $T \geq 2 \theta_{*} T_{+}(\mathcal{Q})$ and $p<2+\sigma$, then in the cylinder $\mathcal{Q}_{1}=\left(x_{0}, t_{0}\right)+$ $Q\left(R, \theta_{*} T / 4\right)$ we have

$$
\operatorname{ess} \operatorname{osc}\left\{u ; \mathcal{Q}_{1}\right\}<(1-\gamma) \operatorname{ess} \operatorname{osc}\{u ; \mathcal{Q}\} \text {. }
$$

Proof of Theorem 1, Let $u$ be a solution of (1.1) in a cylinder $Q=\left(x_{0}, \xi_{0}\right)+Q(R, T)$. Let $N$ be the number from Lemma 11] We construct the sequence of cylinders

$$
Q_{j}=\left(x_{0}, \xi_{0}\right)+Q\left(R_{j}, t_{j}\right), \quad \text { where } R_{j}=\delta^{j} R, \quad \delta \in\left(0, \frac{1}{8}\right),
$$

where $t_{j}$ depends on the behaviour of $u$ in the cylinder $Q_{j-1}$. We can assume without loss of generality that $\delta^{p}<1 /(2 N)$. In each of the cylinders $Q_{j}$ we put

$$
\begin{aligned}
& \mu_{j}=\operatorname{ess} \sup \left\{u ; Q_{j}\right\}, \quad \omega_{j}=\frac{1}{2} \operatorname{ess} \operatorname{osc}\left\{u ; Q_{j}\right\}, \\
& b_{j}=\left(\max \left(\left|\mu_{j}\right|, \omega_{j}\right)\right)^{m-1} \omega_{j}^{p-2}, \quad a_{j}=\frac{2 N R_{j}^{p}}{t_{j}} .
\end{aligned}
$$

It is convenient to bear in mind the relation $2 N b_{j} T_{+}\left(Q_{j}\right)=a_{j} t_{j}$. We define a sequence $t_{j}, j=0,1, \ldots$, as follows. We set $t_{0}=T$. At each step there are three possible options.

I. If $t_{j} \geq 2 N T_{+}\left(Q_{j}\right)$, which is equivalent to $b_{j} \geq a_{j}$, and $\Gamma\left(\mu_{j}, \omega_{j}, R_{j} / 8\right) \leq 1$, then we take $t_{j+1}=T_{+}\left(Q_{j}\right)$. By Lemma 11, in this case we have

$$
\omega_{j+1} \leq(1-\gamma) \omega_{j}, \quad \text { where } \gamma=\gamma(\text { data })>0,
$$

and also

$$
t_{j+1}=T_{+}\left(Q_{j}\right) \leq \delta^{-p} T_{+}\left(Q_{j+1}\right)
$$


II. If $t_{j}<2 N T_{+}\left(Q_{j}\right)$, which is equivalent to $b_{j}<a_{j}$, or $\Gamma\left(\mu_{j}, \omega_{j}, R_{j} / 8\right)>1$, then we take $t_{j+1}=t_{j} /(2 N)$. In this case we have

$$
a_{j+1}=\frac{2 N R_{j+1}^{p}}{t_{j+1}}=2 N \delta^{p} a_{j}
$$

and

$$
t_{j+1}=\frac{t_{j}}{2 N}<T_{+}\left(Q_{j}\right) \leq \delta^{-p} T_{+}\left(Q_{j+1}\right) .
$$

III. It may happen that $t_{j} \geq 2 N T_{+}\left(Q_{j}\right)$ but $\Gamma_{j}=\Gamma\left(\mu_{j}, \omega_{j}, R_{j} / 8\right) \geq 1$. In this case we also set $t_{j+1}=t_{j} /(2 N)$, which gives inequality (5.11). Furthermore, in this case, using the inequality for $\Gamma_{j}$ we obtain the estimate $\omega_{j} \leq C \max \left(R_{j}, R_{j}^{q}\right)$, where $q=(p-1) /(p+m-2) \in(0,1)$. Thus, in all cases,

$$
t_{j} \leq \delta^{-p} T_{+}\left(Q_{j}\right), \quad j \geq 1 .
$$

This immediately implies that

$$
t_{j+1} \geq \min \left(\delta^{p}, \frac{1}{2 N}\right) t_{j} \quad \text { and } \quad b_{j} \leq \frac{a_{j}}{2 N \delta^{p}} \quad \text { for all } j \geq 1 .
$$

We choose

$$
\delta=\min \left(\frac{1}{8},\left(\frac{1}{4 N}\right)^{1 / p}\right) .
$$

We observe that

$$
\omega_{j}^{m+p-3} \leq b_{j} \leq \frac{a_{j}}{2 N \delta^{p}} \text { for } j \geq 1 .
$$

If $k$ is the maximal number in the interval $[1, j]$ at which option III holds, then from (5.9), (5.10) and (5.12) we obtain

$$
\omega_{j} \leq C \alpha^{j-k} \max \left(R_{k}, C R_{k}^{q}\right) \leq C(\max (\alpha, \delta))^{j} \max \left(R^{q}, R\right) .
$$

If, however, among the steps with numbers in $[1, j]$ there are no steps at which option III holds, then we obtain the estimate

$$
\omega_{j} \leq C \alpha^{j} \omega_{1} .
$$

Changing the number $\alpha$ we can assume that in any case,

$$
\omega_{j} \leq C \alpha^{j} \max \left(R^{q}, R, \omega_{1}\right) .
$$

We are now ready to write out an estimate for the modulus of continuity explicitly. We take two points $\left(x_{0}, \xi_{0}\right)$ and $(y, \tau)$ such that $\xi_{0}>\tau$. We can assume that $Q_{0}=$ $\left(x_{0}, \xi_{0}\right)+Q(R, T) \subset \mathcal{Q}$ and $(y, \tau) \in Q_{0}$. Starting from $Q_{0}$ we construct a sequence of cylinders $\left(x_{0}, \xi_{0}\right)+Q\left(\delta^{j} R, t_{j}\right)$ as we did above. We write

$$
\begin{gathered}
\Delta x=\left|y-x_{0}\right|, \quad \Delta t=\left|\tau-\xi_{0}\right|, \\
\mu_{+}=\operatorname{ess} \sup \left\{u ; Q_{0}\right\}, \quad \omega=\frac{1}{2} \operatorname{ess} \operatorname{osc}\left\{u ; Q_{0}\right\}, \quad \mu=\max \left(\left|\mu_{+}\right|, \omega\right) .
\end{gathered}
$$

We observe that

$$
t_{1} \geq \min \left(\frac{T}{2 N}, \mu^{1-m} \omega^{2-p} R^{p}\right) .
$$

Obviously, the point $(y, \tau)$ is contained inside the cylinder $Q_{j}$ if

$$
\frac{\Delta x}{R} \leq \delta^{j} \quad \text { and } \quad \frac{\Delta t}{t_{1}} \leq \Delta t \max \left(\frac{2 N}{T}, \frac{\mu^{m-1} \omega^{p-2}}{R^{p}}\right) \leq \delta^{p(j-1)} .
$$


Thus, $(y, \tau) \in Q_{j}$ for all

$$
j \leq j_{*}=\min \left(\log _{\delta} \frac{\Delta x}{R}, \log _{\delta}\left(\Delta t \max \left(\frac{2 N}{T}, \frac{\mu^{m-1} \omega^{p-2}}{R^{p}}\right)\right)^{1 / p}\right)-2,
$$

and we obtain the estimate

$$
\begin{aligned}
\mid u(y, \tau) & -u\left(x_{0}, \xi_{0}\right) \mid \leq C \alpha^{j_{*}} \omega_{1} \\
& \leq C \max \left(\omega, R^{q}, R\right)\left[\left(\frac{\Delta x}{R}\right)^{p \beta}+\left(\Delta t \max \left(\frac{2 N}{T}, \frac{\mu^{m-1} \omega^{p-2}}{R^{p}}\right)\right)^{\beta}\right],
\end{aligned}
$$

where $\beta=\frac{\log _{\alpha} \delta}{p}$.

\section{$\S 6$. The Harnack Inequality}

We shall need one important technical lemma on 'expansion of positivity' [19].

Lemma 12. Let $u$ be a nonnegative supersolution in the cylinder $Q=\left(x_{0}, t_{0}\right)+B_{8 R} \times$ $[0, T]$. Suppose that for $k>0$,

$$
\nu\left(\left\{u\left(\cdot, t_{0}\right)>k\right\} \cap\left(x_{0}+B_{R}\right)\right) \geq \alpha \nu\left(x_{0}+B_{R}\right), \quad \alpha>0 .
$$

Then there exist positive constants $\gamma_{i}=\gamma_{i}($ data, $\alpha), i=1, \ldots, 4$ such that if $T>$ $\gamma_{1} k^{3-m-p} R^{p}$ and $\Gamma(0, k, R) \leq \gamma_{3}$, then

$$
u(x, t) \geq \gamma_{4} k, \quad\left|x-x_{0}\right|<2 R, \quad t \in\left(\gamma_{1} k^{3-m-p} R^{p}, \gamma_{2} k^{3-m-p} R^{p}\right) .
$$

Proof. We will prove only the first case. We assume that $t_{0}=0$. By Lemma 9 applied with $\mu_{-}=0, \omega=k$, there exist positive constants $\delta, \sigma, \theta_{*}$ depending only on the parameters in the equation such that for any $\xi \in(0,1)$ we have

$$
\nu\left(\{u(\cdot, t)>\delta \xi k\} \cap\left(x_{0}+B_{R}\right)\right) \geq \frac{\alpha}{2} \nu\left(x_{0}+B_{R}\right), \quad \alpha>0,
$$

for all $t<\theta_{*}(\xi k)^{3-m-p} R^{p}$, provided that $\Gamma(0, \xi k, \sigma R) \leq 1$. Using the value

$$
\xi=\min \left\{1,\left(\frac{t}{\theta_{*} k^{3-m-p} R^{p}}\right)^{1 /(3-m-p)}\right\}
$$

we obtain the following estimate:

$$
\begin{gathered}
\nu\left(\{u(\cdot, t)>\delta \varphi(t) k\} \cap\left(x_{0}+B_{R}\right)\right) \geq \frac{\alpha}{2} \nu\left(x_{0}+B_{R}\right), \quad \alpha>0, \\
\varphi(t)= \begin{cases}1, & t \leq \theta_{*} k^{3-m-p} R^{p}, \\
\left(\frac{t}{\theta_{*} k^{3-m-p} R^{p}}\right)^{1 /(3-m-p)}, & t>\theta_{*} k^{3-m-p} R^{p} .\end{cases}
\end{gathered}
$$

We perform the change of variables:

$$
u=\delta \varphi(t) v, \quad \tau=\tau(t)=\int_{0}^{t}(\delta \varphi(s))^{m+p-3} d s .
$$

For $v$ we obtain the equation

$$
\nu(x) \frac{\partial v}{\partial \tau}=\operatorname{div}(\nu(x) \mathbf{B}(x, \tau, v, \nabla v))-\nu(\delta \varphi)^{3-m-p} \varphi_{t} v,
$$

where the flow is

$$
\mathbf{B}(x, \tau, v, \xi)=(\delta \varphi)^{3-m-p} \mathbf{A}(x, t, \delta \varphi(t) v, \delta \varphi(t) \xi), \quad t=t(\tau) .
$$


It is easy to verify that $\mathbf{B}$ satisfies the same structure conditions as $\mathbf{A}$. We observe that

$$
0 \leq-(\delta \varphi)^{3-m-p} \varphi_{t}=\frac{1}{m+p-3} \begin{cases}0, & t \leq \theta_{*} k^{3-m-p} R^{p}, \\ \frac{k^{m+p-3}}{\theta_{*} \delta^{m+p-3} R^{p}}, & t>\theta_{*} k^{3-m-p} R^{p} .\end{cases}
$$

Thus, $v$ is a supersolution of an equation with the same structure as the original, with the same structure constants. From the estimate for $u$ we immediately obtain

$$
\nu\left(\{v(\cdot, \tau)>k\} \cap\left(x_{0}+B_{R}\right)\right) \geq \frac{\alpha}{2} \nu\left(x_{0}+B_{R}\right)
$$

under the condition that

$$
\Gamma(0, \xi k, R) \leq 1, \quad \xi=\xi(\tau)=\min \left\{1,\left(\frac{t(\tau)}{\theta_{*} k^{3-m-p} R^{p}}\right)^{1 /(3-m-p)}\right\} .
$$

Next, we apply the 'telescopic lemma'. We find that for any $\varepsilon>0$ there exists $\delta_{*}=$ $\delta_{*}(\varepsilon)>0$ such that for any $0<\delta_{1}<\delta_{*}(\varepsilon)$, in the cylinder $Q\left(\tau_{0}\right)=\left(x_{0}+B_{4 R}\right) \times\left(\tau_{0}, 2 \tau_{0}\right)$, we have

$$
\nu\left(\left\{v<\delta_{1} k\right\} \cap Q\right)<\varepsilon \nu(Q)
$$

provided that

We take

$$
\tau_{0} \geq\left(\delta_{1} k\right)^{3-m-p}(2 R)^{p}, \quad \Gamma\left(0, \delta_{1} k, 2 R\right) \leq 1
$$

$$
\tau_{0}=\tau_{0}(\varepsilon)=\left(\delta_{1} k\right)^{3-m-p}(2 R)^{p} .
$$

Next, we choose $\varepsilon$ such that in the corresponding cylinder $Q\left(\tau_{0}\right)$ we can apply Lemma 6 with the parameters $a=1 / 2, \mu_{-}=0, \omega=\delta_{1} k$. By our choice of $\tau_{0}$, the parameter satisfies $\theta\left(\tau_{0}, 0, \delta_{1} k, 2 R\right)=1$. Therefore there exists a number $\varepsilon$ such that the inequality

$$
\nu\left(\left\{v<\delta_{1}(\varepsilon) k\right\} \cap Q\left(\tau_{0}\right)\right)<\varepsilon \nu\left(Q\left(\tau_{0}\right)\right)
$$

implies that

$$
v \geq \frac{\delta_{1} k}{2} \quad \text { almost everywhere in }\left(x_{0}+B_{R}\right) \times\left(\frac{3 \tau_{0}}{2}, 2 \tau_{0}\right)
$$

under the condition $\Gamma\left(0, \delta_{1} k, 2 R\right) \leq 1$.

Thus, we can draw the following conclusion: if the conditions on $\Gamma$ given above hold, then there exists a positive number $\delta_{1}=\delta_{1}$ (data) such that $v \geq \delta_{1} k / 2$ almost everywhere in the cylinder $\left(x_{0}+B_{R}\right) \times\left(3 \tau_{0} / 2,2 \tau_{0}\right)$, where $\tau_{0}=\left(\delta_{1} k\right)^{3-m-p}(2 R)^{p}$. We now need to return to the original variable. For $t$ we obtain the formula

$$
t= \begin{cases}\tau, & \tau \leq \theta_{*} k^{3-m-p} R^{p}, \\ \theta_{*} k^{3-m-p} R^{p} \exp \left(\frac{\tau-\theta_{*} k^{3-m-p} R^{p}}{\delta^{m+p-3} k^{3-m-p} \theta_{*} R^{p}}\right), & \tau>\theta_{*} k^{3-m-p} R^{p} .\end{cases}
$$

Next,

$$
\varphi(t(\tau))= \begin{cases}1, & \tau \leq \theta_{*} k^{3-m-p} R^{p}, \\ \exp \left(\frac{\tau-\theta_{*} k^{3-m-p} R^{p}}{(3-m-p) \delta^{m+p-3} k^{3-m-p} \theta_{*} R^{p}}\right), & \tau>\theta_{*} k^{3-m-p} R^{p} .\end{cases}
$$

Returning to the original variables we obtain

$$
u(x, t) \geq \delta \frac{\delta_{1}}{2} k \varphi\left(t\left(2 \tau_{0}\right)\right)=\gamma_{4} k, \quad \gamma_{4}=\gamma_{4}(\text { data })>0,
$$

in the cylinder $\left(x_{0}+B_{2 R}\right) \times\left(t\left(3 \tau_{0} / 2\right), t\left(2 \tau_{0}\right)\right)$. It is easy to see that

$$
t\left(\frac{3 \tau_{0}}{2}\right)=\gamma_{3} k^{3-m-p} R^{p}, \quad t\left(2 \tau_{0}\right)=\gamma_{4} k^{3-m-p} R^{p} .
$$


The conditions on $\Gamma$ can be rewritten in the form

$$
\Gamma\left(0, \gamma_{4} k, R\right) \leq 1
$$

which reduces to $\Gamma(0, k, R)<C$.

Remark. It is easy to see that the constants in this lemma degenerate if the quantity $m+p-3$ is small. In order to stabilize them, we apply the method from [19. We put $T=\theta_{*} k^{3-m-p} R^{p}$. We apply Lemma 10 (taking account of the remark after it) in the cylinder $\left(x_{0}+B_{8 R}\right) \times\left[t_{0}, t_{0}+T\right]$ with $\mu_{-}=0, \omega=k / 2$. Now we set $Q_{1}=$ $\left(x_{0}+B_{4 R}\right) \times\left[t_{0}+T / 2, t_{0}+T\right]$. Then for any $\varepsilon>0$ there exists $\delta=\delta(\varepsilon) \in(0,1)$ and $\sigma=\sigma(\varepsilon)>0$ such that

$$
\nu\left(\left\{u<\frac{\delta k}{2}\right\} \cap Q_{1}\right)<\varepsilon \nu\left(Q_{1}\right), \quad \delta^{3-m-p} \leq 2,
$$

if $m+p<3+\sigma$ and $\Gamma(0, \delta k, 8 R) \leq 1$. Next, we find $\varepsilon$ such that in the cylinder $Q_{1}$ we can apply Lemma 6] with the parameters $\mu_{-}=0, \omega=\delta k / 2, a=1 / 2$. We observe that

$$
\theta\left(\frac{T}{2}, 0, \frac{\delta k}{2}, 4 R\right)+\frac{1}{\theta\left(\frac{T}{2}, 0, \frac{\delta k}{2}, 4 R\right)}<C(\text { data })
$$

Consequently, by choosing a suitable value of $\varepsilon$ in such way that the hypothesis of Lemma 6 is satisfied, we obtain

$$
u(x, t)>\frac{\delta k}{4}, \quad\left|x-x_{0}\right|<2 R, \quad t_{0}+\frac{3}{4} \theta_{*} k^{3-m-p} R^{p} \leq t \leq t_{0}+\theta_{*} k^{3-m-p} R^{p},
$$

where the constants $\delta, \theta_{*}$ are independent of $m, p$ if $m+p<3+\sigma$. Thus, the estimate of Lemma 12 is stable as $m \rightarrow 1, p \rightarrow 2$. Consequently, the estimates of Theorem 2 based on it are also stable as $m \rightarrow 1, p \rightarrow 2$.

Proof of Theorem 2. We now embark on the proof of the Harnack inequality proper. We use the Krylov-Safonov method. We assume without loss of generality that $\left(x_{0}, t_{0}\right)=$ $(0,0)$ and $\lambda=1$. Let $\beta>0$; the exact value of $\beta$ will be determined later. We consider the cylinders

$$
Q(\tau)=B_{(1-\tau) R} \times\left[-\tau k^{3-m-p} R^{p}, 0\right], \quad \tau \in(0,1) .
$$

Let $M(\tau)=\max \{u ; \overline{Q(\tau)}\}$. The solution is bounded in the cylinder $Q(1)$; therefore $M(\tau)<(1-\tau)^{-\beta} k$ for $\tau$ close to 1 . We define $\tau_{0}$ to be the largest root of the equation $M(\tau)=(1-\tau)^{-\beta} k$. Suppose that $M\left(\tau_{0}\right)$ is attained at a point $\left(x_{1}, t_{1}\right) \in \overline{Q\left(\tau_{0}\right)}$. It is easy to see that in the cylinder

$$
Q_{1}=\left(x_{1}, t_{1}\right)+Q\left(\frac{(1-\tau) R}{2}, \frac{(1-\tau) k^{3-m-p} R^{p}}{2}\right)
$$

we have the estimate $u \leq 2^{\beta}(1-\tau)^{-\beta} k$. In the cylinder

$$
Q_{2}=\left(x_{1}, t_{1}\right)+Q\left(\frac{\left(1-\tau_{0}\right) R}{2}, T_{2}\right)
$$

we now apply Lemma 6 with the parameters

$$
\begin{gathered}
\mu_{+}=2^{\beta}\left(1-\tau_{0}\right)^{-\beta} k, \quad \omega=\left(2^{\beta}-2^{-1}\right)\left(1-\tau_{0}\right)^{-\beta} k, \\
T_{2}=T\left(\mu_{+}, \omega, \frac{\left(1-\tau_{0}\right) R}{2}\right), \quad a=\frac{2^{\beta}-3 / 4}{2^{\beta}-1 / 2} .
\end{gathered}
$$

We find that there exists $\varepsilon=\varepsilon(\beta)>0$ such that $u \leq 3\left(1-\tau_{0}\right)^{-\beta} k / 4$ in the cylinder

$$
Q_{3}=\left(x_{1}, t_{1}\right)+Q\left(\frac{\left(1-\tau_{0}\right) R}{4}, \frac{T_{2}}{2}\right)
$$


under the conditions

$$
\nu\left(\left\{u \geq \frac{\left(1-\tau_{0}\right)^{-\beta} k}{2}\right\} \cap Q_{1}\right)<\varepsilon \nu\left(Q_{1}\right), \quad \Gamma\left(\mu_{+}, \omega,\left(1-\tau_{0}\right) R\right) \leq 1 .
$$

Since $u\left(x_{1}, t_{1}\right)=\left(1-\tau_{0}\right)^{-\beta} k$ by hypothesis, we obtain the following:

$$
\nu\left(\left\{u \geq \frac{\left(1-\tau_{0}\right)^{-\beta} k}{2}\right\} \cap Q_{1}\right)>\varepsilon \nu\left(Q_{1}\right) \quad \text { if } \Gamma\left(\mu_{+}, \omega,\left(1-\tau_{0}\right) R\right) \leq 1 .
$$

The last condition reduces to $\Gamma(0, k, R)<\gamma_{2}=\gamma_{2}$ (data).

Consequently, there exists a level $t_{*} \in\left(t_{1}-T_{2}, t_{1}\right)$ such that

$$
\nu\left(\left\{u\left(\cdot, t_{*}\right) \geq \frac{\left(1-\tau_{0}\right)^{-\beta} k}{2}\right\} \cap\left(x_{1}+B_{\left(1-\tau_{0}\right) R / 2}\right)\right)>\varepsilon \nu\left(x_{1}+B_{\left(1-\tau_{0}\right) R / 2}\right), \quad \varepsilon=\varepsilon(\beta) .
$$

Applying Lemma 12 we obtain

$$
\begin{gathered}
u\left(x, t_{2}\right) \geq \frac{\gamma_{0}}{2}\left(1-\tau_{0}\right)^{-\beta} k, \quad\left|x-x_{1}\right|<\left(1-\tau_{0}\right) R, \\
t_{2}=t_{*}+\gamma_{*}\left(\frac{\left(1-\tau_{0}\right)^{-\beta} k}{2}\right)^{3-m-p}\left(\frac{\left(1-\tau_{0}\right) R}{2}\right)^{p},
\end{gathered}
$$

where the constants $\gamma_{0}$ and $\gamma_{*}$ depend on $\beta$. We now apply Lemma 12 repeatedly to obtain

$$
\begin{gathered}
u\left(x, \tau_{N}\right) \geq \frac{\gamma_{0}}{2} \gamma^{N}\left(1-\tau_{0}\right)^{-\beta}, \quad\left|x-x_{1}\right| \leq 2^{N-1}\left(1-\tau_{0}\right) R, \\
\tau_{0}=t_{2}, \quad \tau_{N}=t_{2}+\sum_{j=0}^{N-1} C\left(\gamma_{0} \gamma^{j} k\left(1-\tau_{0}\right)^{-\beta}\right)^{3-m-p}\left(\left(1-\tau_{0}\right) R 2^{j}\right)^{p},
\end{gathered}
$$

where $\gamma, C$ depend only on the parameters of the equation and no longer depend on $\beta$. We choose the least $N$ such that $\left(1-\tau_{0}\right) R 2^{N} \geq 4 R$, and then

$$
2-\log _{2}\left(1-\tau_{0}\right) \leq N<3-\log _{2}\left(1-\tau_{0}\right) .
$$

For this choice of $N$ we have

$$
u\left(x, \tau_{N}\right) \geq k \frac{\gamma_{0} \gamma^{3}}{2}\left(1-\tau_{0}\right)^{\log _{2}(1 / \gamma)}\left(1-\tau_{0}\right)^{-\beta}, \quad x \in B_{2 R} .
$$

For the choice $\beta=\log _{2}(1 / \gamma)$ we obtain the estimate

$$
u\left(x, \tau_{N}\right) \geq \gamma_{4} k, \quad x \in B_{2 R}, \gamma_{4}=\gamma_{4}(\text { data })>0 .
$$

All the steps are valid if

$$
\begin{aligned}
& \Gamma_{1}=\Gamma\left(0, \frac{k}{2}\left(1-\tau_{0}\right)^{-\beta}, \frac{1-\tau_{0}}{2} R\right) \leq 1 \\
& \Gamma_{2}=\Gamma\left(0, \gamma_{0} \gamma^{j}\left(1-\tau_{0}\right)^{-\beta} \frac{k}{2},\left(1-\tau_{0}\right) R 2^{j}\right) \leq 1
\end{aligned}
$$

for $j=0,1, \ldots, N$. These conditions are satisfied for $\Gamma(0, k, R) \leq \gamma_{2}=\gamma_{2}$ (data). Indeed,

$$
\Gamma_{1} \leq 2^{p+m-1} \Gamma(0, k, R), \quad \Gamma_{2} \leq \gamma_{4}^{-p-m} 8^{p} \Gamma(0, k, R) .
$$

Next, we estimate the quantity $\tau_{N}$ :

$$
\tau_{N} \leq t_{*}+C\left(1-\tau_{0}\right)^{p+\beta(3-m-p)} \gamma^{N(3-m-p)} 2^{p N} .
$$

From our choice of $N$ and $\beta$ we have

$$
\left(1-\tau_{0}\right)^{\beta} \gamma^{-N} \leq C \gamma^{-2}, \quad\left(1-\tau_{0}\right) 2^{N} \leq 4 .
$$


Thus, for some $\tau_{N} \in\left[-k^{3-m-p} R^{p}, \gamma_{1} k^{3-m-p} R^{p}\right]$ we have

$$
u\left(x, \tau_{N}\right) \geq \gamma_{4} k, \quad x \in B_{2 R}, \quad \text { where } \gamma_{4}=\gamma_{4}(\text { data })>0 .
$$

Let $\xi \in[0,1]$. We apply Lemma 7 with the parameters $\mu_{-}=0, \omega=\gamma_{4} \xi k, a=1 / 2$ in the cylinder $B_{2 R} \times\left[\tau_{N}, \gamma_{1} k^{3-m-p} R^{p}\right]$. We see that

$$
u\left(x, \gamma_{1} k^{3-m-p} R^{p}\right) \geq \xi \gamma_{4} k, \quad x \in B_{R},
$$

if

$$
\gamma_{1} k^{3-m-p} R^{p}-\tau_{N} \leq C\left(\gamma_{4} \xi k\right)^{3-m-p}(2 R)^{p}, \quad C=C(\text { data })>0
$$

and $\Gamma\left(0, \xi \gamma_{4} k, 2 R\right) \leq 1$. The last condition can be easily transformed to the form

$$
\Gamma(0, k, R) \leq \gamma_{2}=\gamma_{2} \text { (data). }
$$

It is easy to see that (6.1) holds for some $\xi=\xi$ (data) $>0$. Theorem 2 is proved. In order to show the constants are stable as $m \rightarrow 1, p \rightarrow 2$, we need to apply Lemma 12 several times instead of Lemma 7 at the last step.

Remarks. First, for the initial definition of a solution we can manage with the assumption that $\nabla u^{1+\sigma} \in L^{p}(Q ; \nu), \sigma=(m-1) /(p-1)$. But in this case we would have to define a solution more accurately. This question was considered in the papers of Ivanov. Second, it is more natural to use test functions containing $\left(u^{1+\sigma}-k^{1+\sigma}\right)_{ \pm}$. If we follow this path we also obtain a proof in the case when $m<1, m+p-3>-1$.

Next, in the statements of the theorems, in the conditions $\Gamma(\ldots) \leq \gamma$ the constant $\gamma$ can be replaced by an arbitrary positive number; of course the other constants in the statements of the theorems will also change.

We also observe that to prove that $u=u(x, t)$ is Hölder continuous we only need to know that $u$ satisfies the estimates (3.1). So far this has not been sufficient to prove the Harnack inequality. It would be quite interesting to know whether it is possible to obtain the Harnack inequality using only the estimates (3.1).

\section{REFERENCES}

[1] A. V. Ivanov, Hölder estimates for quasilinear doubly degenerate parabolic equations, Zap. Nauchn. Sem. Leningrad. Otdel. Mat. Inst. Steklov. (LOMI) 171 (1989), 70-105; English transl., J. Soviet Math. 56 (1991), no. 2, 2320-2347. MR1031986 (91a:35031)

[2] A. V. Ivanov, Uniform Hölder estimates for generalized solutions of quasilinear parabolic equations that admit double degeneration, Algebra i Analiz 3 (1991), no. 2, 139-179; English transl., St. Petersburg Math. J. 3 (1992), no. 2, 363-403. MR.1137526 (93d:35083)

[3] A. V. Ivanov, The classes $\mathscr{B}_{m, l}$ and Hölder estimates for quasilinear parabolic equations that admit double degeneration, Zap. Nauchn. Sem. S.-Peterburg. Otdel. Mat. Inst. Steklov. (POMI) 197 (1992), 42-70; English transl., J. Math. Sci. 75 (1995), no. 6, 2011-2027. MR1172988(93e:35062)

[4] A. V. Ivanov, Quasilinear parabolic equations that admit double degeneration, Algebra i Analiz 4 (1992), no. 6, 114-130; English transl., St. Petersburg Math. J. 4 (1993), no. 6, 1153-1168. MR.1199637 (94a:35065)

[5] A. V. Ivanov, Hölder estimates for equations of fast diffusion type, Algebra i Analiz 6 (1994), no. 4, 101-142; English transl., St. Petersburg Math. J. 6 (1995), no. 4, 791-825. MR.1304096 (95j:35110)

[6] A. V. Ivanov, Hölder estimates for a natural class of equations of fast diffusion type, Zap. Nauchn. Sem. S.-Peterburg. Otdel. Mat. Inst. Steklov. (POMI) 229 (1995), 29-62; English transl., J. Math. Sci. (New York) 89 (1998), no. 6, 1607-1630. MR1433579 (97m:35123)

[7] A. V. Ivanov, Maximum modulus estimates for generalized solutions to doubly nonlinear parabolic equations, Zap. Nauchn. Sem. S.-Peterburg. Otdel. Mat. Inst. Steklov. (POMI) 221 (1995), 83-113; English transl., J. Math. Sci. (New York) 87 (1997), no. 2, 3322-3342. MR1359751 (96i:35057)

[8] A. V. Ivanov, The regularity theory for $(M, L)$-Laplacian parabolic equation, Zap. Nauchn. Sem. S.-Peterburg. Otdel. Mat. Inst. Steklov. (POMI) 243 (1997), 87-110; English transl., J. Math. Sci. (New York) 99 (2000), no. 1, 854-869. MR1629936 (99k:35104)

[9] V. V. Zhikov, On weighted Sobolev spaces, Mat. Sb. 189 (1998), no. 8, 27-58; English transl., Sb. Math. 189 (1998), no. 7-8, 1139-1170. MR1669639 (2000h:46045) 
[10] O. V. Ladyzhenskaya, V. A. Solonnikov, and N. N. Ural'tseva, Linear and quasi-linear equations of parabolic type, Nauka, Moscow, 1967; English transl., Transl. of Math. Monographs, vol. 23, Amer. Math. Soc., Providence, RI, 1968. MR0241821 (39:3159a)

[11] S. Bonafede and I. I. Skrypnik, On Hölder continuity of solutions of doubly nonlinear parabolic equations with weight, Ukrain. Mat. Zh. 51 (1999), no. 7, 890-903, and Ukrainian Math. J. 51 (2000), no. 7, 996-1012. MR.1727693 (2001f:35057)

[12] F. Chiarenza and M. Frasca, Boundedness for the solutions of a degenerate parabolic equation, Applicable Anal. 17 (1984), no. 4, 243-261. MR757584 (86c:35081)

[13] F. M. Chiarenza and R. P. Serapioni, A Harnack inequality for degenerate parabolic equations, Comm. Partial Differential Equations 9 (1984), no. 8, 719-749. MR748366 (86c:35082)

[14] F. M. Chiarenza and R. P. Serapioni, Degenerate parabolic equations and Harnack inequality, Ann. Mat. Pura Appl. (4) 137 (1984), 139-162. MR772255 (87b:35091)

[15] F. Chiarenza and R. Serapioni, A remark on a Harnack inequality for degenerate parabolic equations, Rend. Sem. Mat. Univ. Padova 73 (1985), 179-190. MR799906 (87a:35110)

[16] F. Chiarenza and R. Serapioni, Pointwise estimates for degenerate parabolic equations, Appl. Anal. 23 (1987), no. 4, 287-299. MR875716 (88c:35060)

[17] T. H. Colding and W. P. Minicozzi, II, Liouville theorems for harmonic sections and applications, Comm. Pure Appl. Math. 51 (1998), no. 2, 113-138. MR1488297 (98m:53053)

[18] E. DiBenedetto, Degenerate parabolic equations, Springer-Verlag, New York, 1993. MR1230384 (94h:35130)

[19] E. DiBenedetto, U. Gianazza, and V. Vespri, Harnack estimates for quasi-linear degenerate parabolic differential equations, Acta Math. 200 (2008), no. 2, 181-209. MR2413134(2009g:35130)

[20] E. DiBenedetto, U. Gianazza, and V. Vespri, Harnack's inequality for degenerate and singular parabolic equations, Springer, New York, 2012. MR2865434

[21] J. Heinonen, T. Kilpeläinen, and O. Martio, Nonlinear potential theory of degenerate elliptic equations, Dover Publications, Mineola, NY, 2006. MR2305115 (2008g:31019)

[22] S. Keith and X. Zhong, The Poincaré inequality is an open ended condition, Ann. Math. (2) 167 (2008), no. 2, 575-599. MR2415381 (2009e:46028)

[23] I. I. Skrypnik, Regularity of solutions of degenerate quasilinear parabolic equations (the weighted case), Ukrain. Mat. Zh. 48 (1996), no. 7, 972-988; English transl., Ukrainian Math. J. 48 (1997), no. 7, 1099-1118. MR 1417254 (97h:35095)

[24] M. Surnachev, A Harnack inequality for weighted degenerate parabolic equations, J. Differential Equations 248 (2010), no. 8, 2092-2129. MR.2595716 (2011b:35257)

[25] V. Vespri, On the local behaviour of solutions of a certain class of doubly nonlinear parabolic equations, Manuscripta Math. 75 (1992), no. 1, 65-80. MR.1156216 (93d:35071)

[26] M. M. Porzio and V. Vespri, Hölder estimates for local solutions of some doubly nonlinear degenerate parabolic equations, J. Differential Equations 103 (1993), no. 1, 146-178. MR.1218742 (94d:35015)

[27] V. Vespri, Harnack type inequalities for solutions of certain doubly nonlinear parabolic equations, J. Math. Anal. Appl. 181 (1994), no. 1, 104-131. MR.1257957 (94k:35171)

Keldysh Institute of Applied Mathematics, Moscow

E-mail address: peitsche@yandex.ru

Translated by E. KHUKHRO

Originally published in Russian 